\title{
Algorithms for magnetic resonance imaging in radiotherapy
}

\author{
Jens Sjölund
}

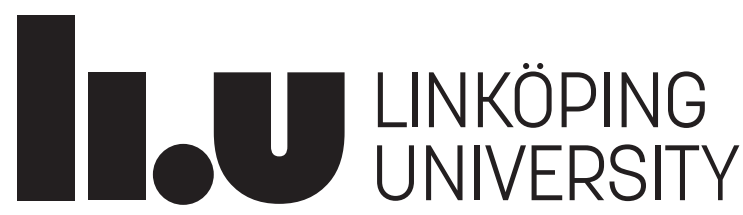

Linköping University

Department of Biomedical Engineering

Division of Biomedical Engineering

SE-581 83 Linköping, Sweden

Linköping 2018 
Edition 1:1

(C) Jens Sjölund, 2018

ISBN 978-91-7685-363-4

ISSN 0345-7524

URL http://urn.kb.se/resolve?urn=urn:nbn:se:liu:diva-144351

Published articles have been reprinted with permission from the respective copyright holder.

Typeset using $\mathrm{X}_{\mathrm{H}} \mathrm{T}_{\mathrm{E}} \mathrm{X}$

Printed by LiU-Tryck, Linköping 2018 


\section{POPULÄRVETENSKAPLIG SAMMANFATTNING}

Cancer. Blotta ordet tycks skrämmande. I snitt drabbas en av tre svenskar av cancer och i takt med att befolkningen åldras förväntas allt fler insjukna. Dessbättre finns numera många effektiva behandlingsformer. Kirurgi är vanligast, men strålbehandling spås få en allt viktigare roll både i kombination med och som alternativ till kirurgi. Till grund för mer effektiv och skonsam strålbehandling ligger ofta nya möjligheter att på ett precist sätt avbilda de berörda kroppsdelarna. Denna avhandling har det långsiktiga målet att leda till förbättrad strålbehandling av cancer genom nya och förbättrade användningar av medicinska bilder tagna med magnetresonanstomografi (eng. MRI). Förenklat kan man dela in planeringen av en strålbehandling i att bestämma vad som ska behandlas och hur det ska behandlas. Båda dessa frågor berörs i avhandlingen.

En trend inom strålbehandling är att ersätta skiktröntgen (eng. CT) med MR som utgångspunkt för planeringen av en strålbehandling. Detta är önskvärt av flera skäl, men framförallt för att den högre bildkvalitéten i MR leder till mer effektiva behandlingar med färre biverkningar. För att simulera var strålningen kommer att absorberas i patienten krävs dock information som inte går att utläsa direkt från MR bilder. I avhandlingen beskrivs två metoder som angriper detta problem. Den första använder artificiell intelligens för att identifiera skallbenet (utöver huvudets kontur). Den andra metoden bygger på att man har en databas av bilder från tidigare patienter vars MR-bilder man får att efterlikna MR-bilden för den aktuella patienten. På så vis kan man också överföra förslag på hur motsvarande CT-bilder bör se ut, så att man kan generera en så kallad pseudo-CT som kan användas för vidare dosberäkningar.

När det gäller att identifiera vad som ska behandlas utgår vi från det faktum att tumörvävnad har en annan struktur än frisk vävnad. Det gör att de vattenmolekyler som finns i de olika vävnadstyperna — och som på grund av värmen befinner sig i ständig rörelse - följer olika rörelsemönster. Detta går att mäta med hjälp av MR. Det benämns då som diffusions-MR, eftersom diffusion är den vetenskapliga termen för slumpmässig värmerörelse. I avhandlingen beskriver vi nya sätt att utföra mätningar med diffusions-MR, och fokuserar mer specifikt på hur man kan göra det möjligt att utföra dessa mätningar på MR-system som används i kliniskt bruk.

Slutligen analyserar vi också hur mätningar gjorda med diffusions-MR används för att anpassa olika modeller. Vi beskriver en ny metod som gör det möjligt att använda en sorts avancerad modell med betydligt färre datapunkter än normalt. Dessutom lägger vi särskild tonvikt på hur man kan få ut mått på osäkerheten för en populär klass av modeller, samt hur denna osäkerhet fortplantar sig till olika så kallade biomarkörer som kan användas vid behandling. 


\begin{abstract}
Radiotherapy plays an increasingly important role in cancer treatment, and medical imaging plays an increasingly important role in radiotherapy. Magnetic resonance imaging (MRI) is poised to be a major component in the development towards more effective radiotherapy treatments with fewer side effects. This thesis attempts to contribute in realizing this potential.
\end{abstract}

Radiotherapy planning requires simulation of radiation transport. The necessary physical properties are typically derived from $\mathrm{CT}$ images, but in some cases only MR images are available. In such a case, a crude but common approach is to approximate all tissue properties as equivalent to those of water. In this thesis we propose two methods to improve upon this approximation. The first uses a machine learning approach to automatically identify bone tissue in MR. The second, which we refer to as atlas-based regression, can be used to generate a realistic, patient-specific, pseudo-CT directly from anatomical MR images. Atlas-based regression uses deformable registration to estimate a pseudo-CT of a new patient based on a database of aligned MR and CT pairs.

Cancerous tissue has a different structure from normal tissue. This affects molecular diffusion, which can be measured using MRI. The prototypical diffusion encoding sequence has recently been challenged with the introduction of more general gradient waveforms. One such example is diffusional variance decomposition (DIVIDE), which allows non-invasive mapping of parameters that reflect variable cell eccentricity and density in brain tumors. To take full advantage of such more general gradient waveforms it is, however, imperative to respect the constraints imposed by the hardware while at the same time maximizing the diffusion encoding strength. In this thesis we formulate this as a constrained optimization problem that is easily adaptable to various hardware constraints. We demonstrate that, by using the optimized gradient waveforms, it is technically feasible to perform whole-brain diffusional variance decomposition at clinical MRI systems with varying performance.

The last part of the thesis is devoted to estimation of diffusion MRI models from measurements. We show that, by using a machine learning framework called Gaussian processes, it is possible to perform diffusion spectrum imaging using far fewer measurements than ordinarily required. This has the potential of making diffusion spectrum imaging feasible even though the acquisition time is limited. A key property of Gaussian processes, which is a probabilistic model, is that it comes with a rigorous way of reasoning about uncertainty. This is pursued further in the last paper, in which we propose a Bayesian reinterpretation of several of the most popular models for diffusion MRI. Thanks to the Bayesian interpretation it possible to quantify the uncertainty in any property derived from these models. We expect this will be broadly useful, in particular in group analyses and in cases when the uncertainty is large. 


\section{Acknowledgments}

In popular culture, research is often depicted as a lonely endeavor. Let me tell you that it is not - at least not in my case. There are a number of people to whom I would like to express my gratitude.

Many thanks goes to my main supervisor Hans Knutsson, for being an endless source of inspiration on time management and rock ' $\mathrm{n}$ ' roll research. Thanks to Anders Eklund, the (formerly) unsung hero, and Mats Andersson, for allowing me to put forward this thesis despite never winning a set. Thanks to Evren and Cem, for generously sharing your insights on all things physical, and for proofreading this thesis. I would like to thank Filip Szczepankiewicz for a wonderful collaboration, and Carl-Fredrik Westin for your hospitality and for introducing me to your team. Thanks also to Hebbe, Xuan, Olivier, Daniel and everyone else at Linköping university whom I've had the fortune of working with.

My sincere thanks goes out to my opponent, Dr. Aasa Feragen, and to the examination committee, Dr. Stefan Skare, Prof. Fredrik Kahl, Prof. Crister Ceberg and Prof. Göran Salerud for taking the time to actually read the whole thesis.

The Swedish Research Council is acknowledged for funding part of this research as an industrial $\mathrm{PhD}$ project together with Elekta, where I am fortunate to work. The Center for Medical Image Science and Visualization (CMIV) at Linköping University is acknowledged for provision of financial support and access to leading edge research infrastructure.

I would like to extend my gratitude to the research and physics group at Elekta, for their expertise, curiosity and friendship; to Jonas Gårding, for playing the long game in a time of quarterly reports and to Håkan Nordström, my informal mentor.

Thanks to LG and Madde, for making me feel as part of the family.

My warmest appreciations go to my mother Eva, for inspiring me to help others, selflessly, and to my brother Joakim, for being a rock. I am forever grateful to Tomas Sjölund and Barbro Gustafsson, for making me who I am. You are with me, always.

And finally, to Maria, for sharing laughter and sorrow, and to Lo, for being a constant reminder of what really matters. 


\section{Contents}

Abstract iii

Acknowledgments v v

Contents vi

List of Figures viii

List of Tables ix

1 Introduction 1

1.1 Aim . . . . . . . . . . . . . . . . . . 1

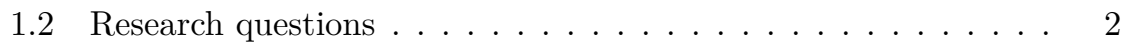

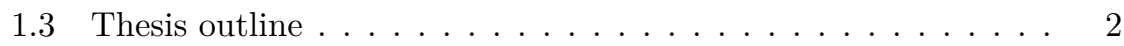

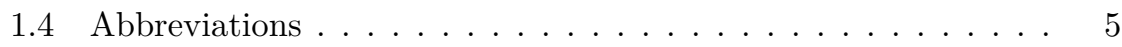

$\begin{array}{lll}2 \text { Radiotherapy } & 7\end{array}$

2.1 Radiobiology . . . . . . . . . . . . . . . . . . . 7

2.2 Radiotherapv modalities ... . . . . . . . . . . . . . 10

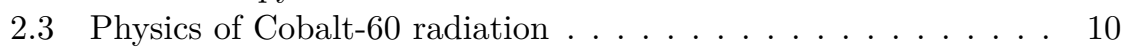

2.4 Treatment planning $\ldots \ldots \ldots \ldots \ldots \ldots \ldots$

3 Magnetic resonance imaging $\quad 15$

3.1 The origin of the MR signal $\ldots \ldots \ldots \ldots \ldots \ldots$

3.2 Excitation and relaxation . . . . . . . . . . . . . . 18

3.3 Pulse sequences . . . . . . . . . . . . . . . . . . . . 21

3.4 Signal localization $\ldots \ldots \ldots \ldots \ldots \ldots \ldots$

4 Diffusion MRI 27

4.1 Diffusion . . . . . . . . . . . . . . . . . . . . . . 27

4.2 Measuring diffusion with MRI $\ldots \ldots \ldots \ldots \ldots \ldots$

4.3 The Stejskal-Tanner experiment . . . . . . . . . . . . . . . . 35

4.4 Diffusional variance decomposition (DIVIDE) . . . . . . . 38 
5 Probabilistic modelling 41

5.1 Regression models in diffusion MRI . . . . . . . . . . . . 41

5.2 Bavesian linear regression . . . . . . . . . . . . . . . . . 42

5.3 Gaussian processes . . . . . . . . . . . . . . . . . . . . 44

5.4 The connection between Bayesian linear regression and Gaus-

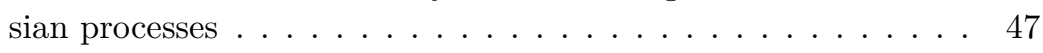

$\begin{array}{lll}6 \text { Summary of papers } & 49\end{array}$

Bibliography 53

\begin{tabular}{ll}
\hline Paper I & 67
\end{tabular}

\begin{tabular}{ll}
\hline Paper II & 75
\end{tabular}

\begin{tabular}{|ll}
\hline Paper III & 93
\end{tabular}

\begin{tabular}{ll}
\hline Paper IV & 107
\end{tabular}

\begin{tabular}{ll}
\hline Paper V & 123
\end{tabular}

\begin{tabular}{ll}
\hline Paper VI & 131
\end{tabular} 


\section{List of Figures}

2.1 Ionizing radiation causes DNA damage. . . . . . . . . . . . . 8

2.2 The Leksell Gamma Knife ${ }^{\circledR}$. . . . . . . . . . . . . . . . . . . . . . 11

2.3 Radioactive decay of Cobalt-60. . . . . . . . . . . . . . 11

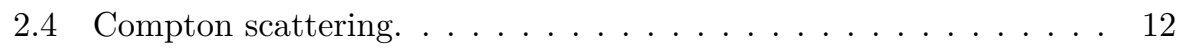

3.1 Precession of the magnetic moment. . . . . . . . . . . 18

3.2 Examples of different MRI contrasts. . . . . . . . . . . . . . 20

3.3 Different ways of sampling k-space. . . . . . . . . . . . . 25

4.1 The Stejskal-Tanner sequence - the mainstay of diffusion MRI. . . 35 


\section{List of Tables}

$3.1 \quad$ Typical values of $T_{1}$ and $T_{2}$ for some different tissue types in a 

"It's easier to resist at the beginning than at the end."

Leonardo da Vinci (1452-1519)

Cancer. The word itself seems frightening. One in three Swedes is expected to develop cancer at some point [81]. Yet, there is hope. Taken over all cancer types, the 5-year survival ratio relative to the general population is about $65 \%$ - and improving [111, 112]. Surgery is the most common treatment form, but radiotherapy plays an increasingly important role [64, 81].

Improvements in radiotherapy, in turn, go hand in hand with those in medical imaging. Today's radiotherapy workflow is based on X-ray computed tomography (CT). Magnetic resonance imaging (MRI) is, however, rapidly being introduced due to its superior soft tissue contrast, the abscence of ionizing radiation, and the possibility of imaging physiological processes [79]. Although MRI is likely to-by and large -improve radiotherapy, it is not without its own set of uncertainties. It is my humble hope that this thesis will contribute to reducing these uncertainties.

\subsection{Aim}

The aim of this thesis is to increase the utility of magnetic resonance imaging in radiotherapy. This aim can be subdivided into three parts. First, 
to facilitate MRI-centered workflows by alleviating the need for CT imaging to perform dose calculations. Second, to further increase the sensitivity of MRI in the detection and classification of tumors, by supporting foundational developments that will ultimately lead to the development of new biomarkers. Third, to promote a prudent usage of biomarkers through uncertainty quantification.

\subsection{Research questions}

1. Is it possible, using only MR images, to derive a substitute to CT images for use in radiotherapy?

2. How can we make diffusion MRI with continuous gradient waveforms viable on clinical MR systems?

3. Is it possible to develop or reinterpret existing diffusion MRI models in a way that allows uncertainty quantification?

\subsection{Thesis outline}

This thesis consists of a background part followed by six research papers. The background part, in turn, consists of four chapters. Chapter 2 provides a general background on radiotherapy: from the principles of how ionizing radiation interacts with biological matter, to how treatment plans are designed. Chapter 3 covers the physics behind the magnetic resonance phenomena and how it can be used to generate images with various contrasts. Chapter 4 describes how magnetic resonance can be used to probe translational motion on the microscopic level. Finally, chapter 5 describes regression models and, in particular, the connection between Bayesian linear regression and Gaussian processes. 


\section{Publications}

I. Jens Sjölund, Andreas Eriksson Järliden, Mats Andersson, Hans Knutsson, and Håkan Nordström. "Skull Segmentation in MRI by a Support Vector Machine Combining Local and Global Features". In: 22nd International Conference on Pattern Recognition (ICPR). IEEE. 2014, pp. 3274-3279. DOI: 10.1109/ICPR.2014.564

II. Jens Sjölund, Daniel Forsberg, Mats Andersson, and Hans Knutsson. "Generating patient specific pseudo-CT of the head from MR using atlas-based regression". In: Physics in Medicine and Biology 60.2 (2015), pp. 825-839. DOI: 10.1088/0031-9155/60/2/825

III. Jens Sjölund, Filip Szczepankiewicz, Markus Nilsson, Daniel Topgaard, Carl-Fredrik Westin, and Hans Knutsson. "Constrained optimization of gradient waveforms for generalized diffusion encoding". In: Journal of Magnetic Resonance 261 (2015), pp. 157-168. DOI: 10.1016/j.jmr. 2015.10 .012

IV. Filip Szczepankiewicz, Jens Sjölund, Freddy Ståhlberg, Jimmy Lätt, and Markus Nilsson. "Whole-brain diffusional variance decomposition (DIVIDE): Demonstration of technical feasibility at clinical MRI systems". In: arXiv preprint arXiv:1612.06741 (2016). In preparation

V. Jens Sjölund, Anders Eklund, Evren Özarslan, and Hans Knutsson. "Gaussian process regression can turn non-uniform and undersampled diffusion MRI data into diffusion spectrum imaging". In: 14th International Symposium on Biomedical Imaging (ISBI). IEEE. 2017, pp. 778782. DOI: $10.1109 /$ ISBI. 2017.7950634

VI. Jens Sjölund, Anders Eklund, Evren Özarslan, Magnus Herberthson, Maria Bånkestad, and Hans Knutsson. "Bayesian uncertainty quantification in linear models for diffusion MRI". in: arXiv preprint arXiv:1711.06002 (2017). Submitted for publication

\section{Publications not included in thesis}

VII. Johanna Skarpman Munter and Jens Sjölund. "Dose-volume histogram prediction using density estimation". In: Physics in medicine and biology 60.17 (2015), pp. 6923-6936. DOI: 10.1088/0031-9155/60/17/6923

VIII. Jens Sjölund, Markus Nilsson, Daniel Topgaard, Carl-Fredrik Westin, and Hans Knutsson. "Constrained optimization of gradient waveforms for isotropic diffusion encoding". In: Proceedings of the International Society of Magnetic Resonance in Medicine. 2015 
IX. Filip Szczepankiewicz, Jens Sjölund, Freddy Ståhlberg, Jimmy Lätt, and Markus Nilsson. "Whole-brain diffusional variance decomposition (DIVIDE) in 8 minutes: Technical feasibility at 1.5, 3, and 7 T". in: Proceedings of the International Society of Magnetic Resonance in Medicine. 2017

\section{Patent applications}

X. Jens Sjölund. Method And System For Calibration. Patent Application PCT/EP2014/057659. Priority date 2014-04-15

XI. Jens Sjölund and Xiao Han. System And Method For Automatic Treatment Planning. United States Patent Application US20150367145 A1. Priority date 2014-06-18

XII. Jens Sjölund. Radiotherapy planning systems. Patent Application PCT/EP2016/079560. Priority date 2015-12-04

XIII. Jens Sjölund. Systems and methods for optimized treatment planning. United States Patent Application US20170177812 A1. Priority date 2015-12-25

XIV. Jens Sjölund and Håkan Nordström. Convex inverse planning method. United States Patent Application US 2017xxxxxxx A1. Priority date 2017-04-28 


\subsection{Abbreviations}

This table lists some of the abbreviations that are used in this thesis, along with their meanings.

\begin{tabular}{|c|c|}
\hline ADC & Apparent Diffusion Coefficient \\
\hline $\mathrm{CPU}$ & Central Processing Unit \\
\hline CSD & Constrained Spherical Deconvolution \\
\hline $\mathrm{CSF}$ & Cerebrospinal Fluid \\
\hline $\mathrm{CT}$ & Computed Tomography \\
\hline DIVIDE & DIffusional VarIance DEcomposition \\
\hline dMRI & Diffusion Magnetic Resonance Imaging \\
\hline DTI & Diffusion Tensor Imaging \\
\hline EAP & Ensemble Averaged Propagator \\
\hline FA & Fractional Anisotropy \\
\hline FFT & Fast Fourier Transform \\
\hline FID & Free Induction Decay \\
\hline GE & Gradient Echo \\
\hline GM & Gray Matter \\
\hline GPU & Graphics Processing Unit \\
\hline GP & Gaussian Process \\
\hline MD & Mean Diffusivity \\
\hline MR & Magnetic Resonance \\
\hline MRI & Magnetic Resonance Imaging \\
\hline NMR & Nuclear Magnetic Resonance \\
\hline $\mathrm{PD}$ & Proton Density \\
\hline QTI & Q-space Trajectory Imaging \\
\hline PFG & Pulsed Field Gradient \\
\hline $\mathrm{RF}$ & Radio Frequency \\
\hline RTOP & Return To Origin Probability \\
\hline $\mathrm{SE}$ & Spin Echo \\
\hline SBRT & Stereotactic Body Radiation Therapy \\
\hline SNR & Signal to Noise Ratio \\
\hline SQP & Sequential Quadratic Programming \\
\hline SRS & Stereotactic Radiosurgery \\
\hline SVM & Support Vector Machine \\
\hline WM & White Matter \\
\hline
\end{tabular}





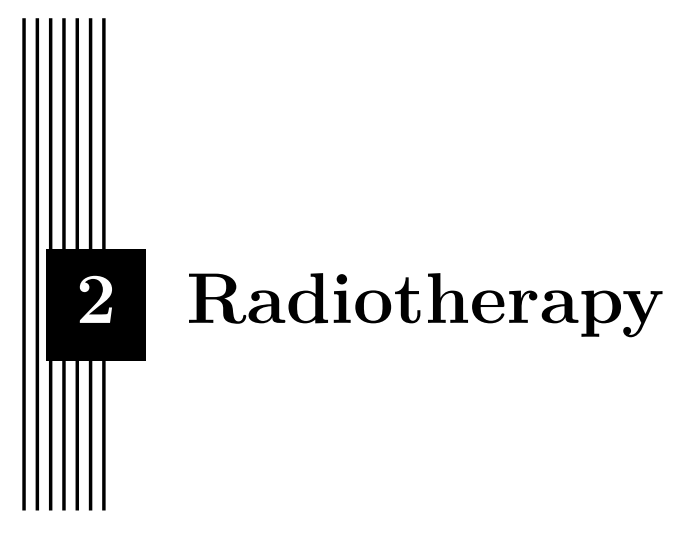

"The dose makes the poison"

Paracelsus (1493-1541)

Radiotherapy is the therapeutic use of ionizing radiation, often with the intent to kill a tumor while minimizing damage to healthy surrounding tissue.

In 2003, the Swedish Council on Technology Assessment in Health Care (SBU) evaluated the role of radiotherapy in the treatment of tumors [81]. It was concluded that radiotherapy has an important role in the cure and palliation of many cancer types - contributing to cure in about $40 \%$ of the patients. Radiotherapy is also a highly cost-effective treatment [68]. We are currently witnessing rapid technological development in every aspect of radiotherapy [53, 78, 79]. This is likely to to further increase the importance of radiotherapy in the future [108].

This chapter provides a general background on radiotherapy: from the principles of how ionizing radiation interacts with biological matter to how treatment plans are designed.

\subsection{Radiobiology}

This section will provide an overview of how ionizing radiation interacts with living tissue; a field called radiobiology. The presentation that follows cover the predominant view in radiobiology, although it is an active field of research 

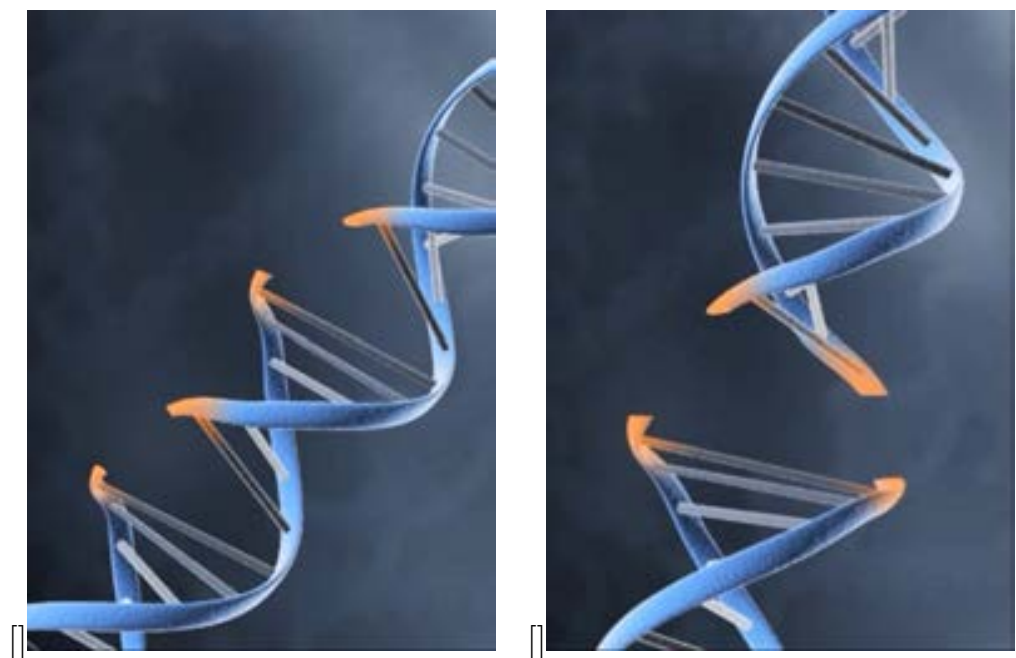

Figure 2.1: Ionizing radiation causes single- and double-strand breaks in the DNA. (OElekta

where studies emphasizing different biological mechanisms have received significant attention [30, 76].

\section{Cellular damage caused by ionizing radiation}

Ionizing radiation primarily affects the cell by causing single- or double-strand breaks in its DNA, see figure 2.1. A single-strand break is when one strand of the DNA-helix is destroyed. Since the opposing strand can be used as a template, single-strand breaks are easy to repair. This is not possible with double-strand breaks, which are more difficult to repair. Failure to repair DNA damage may stop the cell's normal function and proliferation capacity, although it can take months until cell death actually occurs.

Different types of radiation interact with matter in different ways. A heavy charged particle moves in a straight track with dense interactions, while a photon's interactions are sparse and often scatters the photon in different directions. Because of their large mass and electric charge, protons and heavier ions are much more likely to cause a double-strand break compared with the sparsely ionizing photon, and therefore ions are more efficient in killing cells than photons. The larger the mass of the ion, the more efficiently cells are killed.

Although radiation energy is deposited in discrete events on a microscopic level, it is convenient to describe it using the local average of the energy depositions on a macroscopic scale. This is done with the quantity absorbed dose - or simply dose - that describes the average energy absorbed by a mass 
of e.g. tissue or water. Absorbed dose is expressed in the unit of Gray (Gy). One Gy is the absorption of one Joule $(\mathrm{J})$ per kilogram $(\mathrm{kg})$ of mass.

Gamma radiation is another name for highly energetic photons. As a rule of thumb, one third of the DNA damage caused by gamma radiation is from direct photon interaction, whereas the remaining two-thirds of the damage is caused by free radicals induced by the radiation. In the case of gamma radiation, one Gy of absorbed dose in tissue will cause approximately 1000 single-strand breaks, 40 double-strand breaks and a large amount of other aberrations in the DNA [119]. Damage in the double helix of the DNA may at subsequent cell divisions lead to cell death.

\section{The five Rs of radiobiology}

Almost a century of research on the biological basis of radiotherapy has revealed five factors that are critical in determining the net effect of radiotherapy on tumors [12, 14, 105, 128]. They are as follows:

Repair Cellular repair processes are always active. Their effectiveness means that if the dose rate (dose per unit time) decreases, the total dose required to achieve the same probability of cell kill increases.

Redistribution The life cycle of a dividing cell consists of four phases [5] with different radiosensitivity.

Repopulation Cells proliferate over time, but the rate of proliferation varies widely depending on the cell type. In particular, cancer is characterized by uncontrolled cell growth.

Reoxygenation The presence of molecular oxygen increases DNA damage through the formation of oxygen free radicals [38]. Moreover, hypoxia induces proteome and genome changes that may have a substantial impact on radioresistance [41, 117]. Because of these effects, about three times as large radiation dose is required to achieve the same level of cell kill under hypoxic conditions as under normal conditions [33].

Radiosensitivity There is an inherent difference in radiosensitivity between different cell types [19, 27]. Although not universally true, tumor cells are more radiosensitive than the majority of body cells.

Each of these effects can work both ways. For example, if a given dose of radiation is divided into a number of fractions, then redistribution and reoxygenation may over time redistribute surviving tumor cells into more sensitive states, increasing overall cell kill. On the other hand, because of repair and repopulation, cells recover and proliferate, increasing overall cell survival. Modern radiotherapy strives to manipulate these effects to maximize tumor cell kill while avoiding normal tissue toxicities. 


\subsection{Radiotherapy modalities}

Radiotherapy can be carried out with a radiation source placed either inside the body (brachytherapy) or outside the body (external beam radiotherapy).

In brachytherapy, radioactive sources are placed temporarily or permanently. Temporary sources are usually placed with the help of a hollow tube, called an applicator, positioned close to or inside the target.

In external beam radiotherapy, the patient typically lies on a couch while an external source directs an energetic beam at the target. External beam radiotherapy with gamma radiation (photons) is, by far, the most common type of radiotherapy. In particle therapy, another form of external beam radiotherapy, beams of energetic protons, neutrons or heavier ions are used.

Stereotactic treatments refer to external beam radiotherapy where large doses are delivered in a few fractions with exceptionally high targeting accuracy. A distinction is often made between stereotactic radiosurgery (SRS), where the target is in the brain or spine, and stereotactic body radiation therapy (SBRT) where the target is elsewhere in the body. This thesis was written mainly with applications to stereotactic radiosurgery in mind.

\section{Stereotactic radiosurgery}

In 1951, the concept of radiosurgery was introduced by the Swedish neurosurgeon Lars Leksell as a "single high dose fraction of radiation, stereotactically directed to an intracranial region of interest" [56]. Although its scope has broadened over the years, the crucial role of precise targeting in stereotactic radiosurgery remains the same.

The heritage of Lars Leksell lives on in the Leksell Gamma Knife ${ }^{\circledR}$, a machine for SRS that irradiates cerebral targets with narrow high-energy beams of gamma radiation from many directions, see figure 2.2. At the beams intersection, energy from all beams is delivered to the cells. Outside that region, the radiation dose decreases rapidly. This, together with the exceptional targeting accuracy of such a SRS system, enables the delivery of a large, and yet localized, dose to the target while minimizing dose to the surrounding normal tissue.

Although there is strong clinical evidence for the effectiveness of stereotactic radiosurgery [7, 58, 59, 60], its radiobiological effects are not completely understood. There's an ongoing debate whether additional effects to the ones described in section 2.1 come into play for SRS and SBRT [11, 14, 50, 72].

\subsection{Physics of Cobalt-60 radiation}

In the Gamma Knife, radiation is produced by the radioactive decay of Cobalt$60\left({ }^{60} \mathrm{Co}\right)$ sources. Radioactivity is the process whereby unstable atoms release their energy, generally by emitting massive particles or photons, in order to 


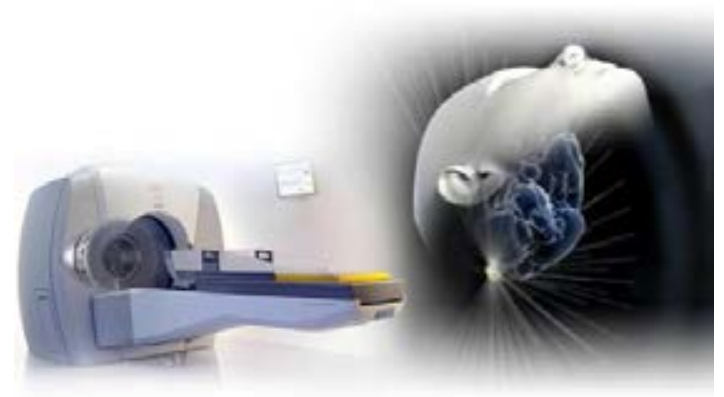

Figure 2.2: The Leksell Gamma Knife ${ }^{\circledR}$ is a machine for stereotactic radiosurgery that irradiates cerebral targets with narrow high-energy beams of gamma radiation from many directions. OElekta

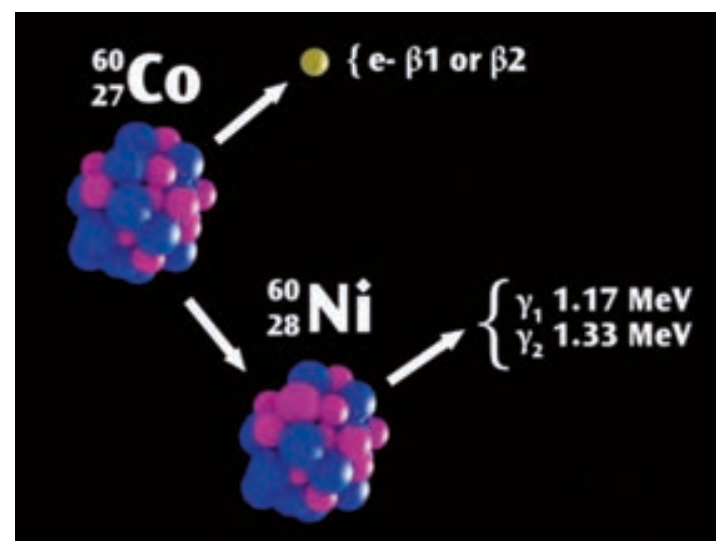

Figure 2.3: Radioactive decay of Cobalt-60, which is used in the Gamma Knife. CElekta

reach its stable, non-radioactive, state. This transition from an unstable atom to the final stable state can include several steps. At each such step energy is radiated.

Figure 2.3 depicts how ${ }^{60}$ Co releases its excess energy. One of the neutrons of the ${ }^{60} \mathrm{Co}$ nucleus transforms, through weak interaction, into a proton and an electron. The electron is instantly emitted by the nucleus, but never reaches the patient since it is absorbed by matter in the radiation source. By this process the ${ }^{60} \mathrm{Co}$ nucleus transforms into a new element, Nickel-60 $\left({ }^{60} \mathrm{Ni}\right)$. The nucleus of ${ }^{60} \mathrm{Ni}$ instantly emits two gamma photons with energies of 1.17 and $1.33 \mathrm{MeV}$, respectively.

Each radionuclide has a characteristic half-life. The half-life of ${ }^{60} \mathrm{Co}$ is 5.27 years. In practice this means that after about five years the radioactivity is only half of the initial radioactivity and the irradiation time required 


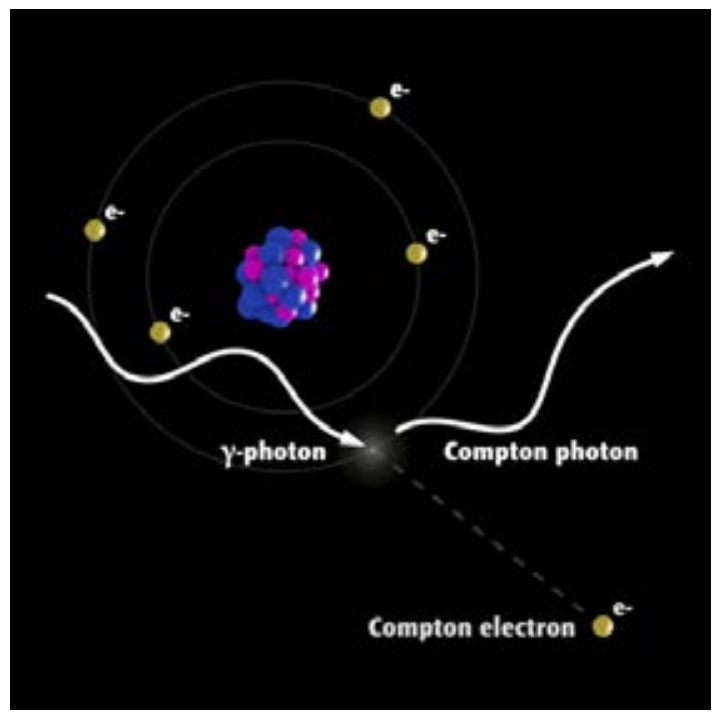

Figure 2.4: Gamma radiation used in external beam radiotherapy mainly interacts with tissue via Compton scattering, a process where the incoming photon scatters inelastically against an electron. OElekta

to achieve a certain dose is doubled. Treatment times are prolonged accordingly, meaning that fewer patients can be treated per day. Thus, the sources eventually need to be replaced.

\section{Energy deposition in photon beams}

In conventional external beam radiotherapy as well as stereotactic radiosurgery with the Gamma Knife, the radiation consists of photons in the $\mathrm{MeV}$ range. In this energy range, the interaction with tissue occurs mainly via a process called Compton scattering, shown in figure 2.4. In short, Compton scattering is an interaction in which the incoming photon scatters inelastically against an electron. The energy imparted to the electron makes it recoil and it is thereby ejected from the atom. The remaining part of the energy is emitted as a scattered photon in a different direction than the electron, such that the overall momentum is conserved.

The recoiling electron ionizes and excites atoms along its trajectory until its kinetic energy is expended. The mean free path of the electron is considerably shorter than that of the scattered photon, which means its energy is deposited more locally. Therefore, it is common to make a distinction between primary dose, deposited by the electron close to the place of interaction, and secondary dose due to the scattered photon, which also eventually deposits its energy through electron interactions. 


\subsection{Treatment planning}

In radiotherapy, treatment planning is the - almost entirely computer basedprocess in which a team of medical personnel develops a patient-specific radiotherapy treatment plan. Typically, medical images acquired with Computed Tomography (CT) or magnetic resonance imaging (MRI) are used to create a patient model in which additional information such as the treatment target and the spatial distribution of the dose is overlaid.

Broadly speaking, treatment planning consists of two main steps: deciding what to treat and how to treat it. The target, and organs at risk, are specified by delineating them on a primary set of images. Often supplementary sets of images are also used. Next, a decision is made on the desired dose to the target and possibly also what doses that can be tolerated in the organs at risk. The clinician then attempts to realize this plan, either by manually specifying the machine parameters (forward planning) or using an optimization procedure (inverse planning). This process is interlaced with simulations of how the dose will be deposited given the current machine parameters.

Today, CT is the mainstay of the radiotherapy workflow [79]. However, the superior soft tissue contrast of MRI already makes it the preferred modality for a number of anatomical locations such as the brain, abdomen and pelvis. This is why stereotactic radiosurgery - contrary to general radiotherapy - has a tradition of planning primarily on MR images. There is currently a strong movement towards MR based radiotherapy [79], fueled also by the possibility of using MRI for so called functional imaging, i.e. imaging physiology instead of anatomy.

One hurdle that an entirely MR based workflow has to overcome-and which is the motivation of papers I and $\mathrm{II}$ is how to perform dose calculations when a CT is not available. To understand why this is an issue, the next section describes how CT based dose calculations are done.

\section{Dose calculations}

The dose quantifies the radiation energy delivered to the tissue. As described in section 2.1, the dose directly relates to the survival probability of irradiated cells and so plays a key role in treatment planning. The spatial distribution of the dose, given a set of machine parameters, can be estimated by a range of different algorithms. The choice of algorithm involves a tradeoff between computational speed and accuracy. The simplest (and fastest) dose calculation algorithms approximate all tissue as water-a reasonably good approximation for soft tissue in homogeneous regions [17, 74] but less so in heterogeneous regions $[129$.

To correct for tissue heterogeneity, more accurate dose calculation algorithms use the Hounsfield units (HU) provided by CT. As described in section 2.3, the incoming photons mainly interact with electrons in the tissue atoms 
through Compton scattering. In the energy range of radiotherapy, the linear attenuation coefficient for Compton scattering is almost completely dependent on the electron density [28, 86]. For MRI, on the other hand, there is no relation between image intensities and electron density.

Hounsfield units have two calibration points, water and air, which are set to 0 and $-1000 \mathrm{HU}$. Conversion from Hounsfield units to electron density can be done using semi-empirical formulas [28, 49] or via a tissue look up table $[73,113]$. Proton and ion beams interact with tissue in a variety of ways, each with a different relationship to the material characteristics obtained from CT [63]. This implies that for protons and ions, the ability to precisely define tissues based on CT scans has an immediate impact on the accuracy of dose calculations [45, 84].

There are two major types of dose calculation algorithms that take tissue heterogeneity into account: superposition-based [3, 90] and Monte Carlobased [87]. In all of the approaches using heterogeneity corrections, the computationally most intensive part is the simulation of electron transport.

Superposition-based algorithms convolve the energy released by a photon with pre-computed energy deposition kernels that are scaled with density. In a pencil beam algorithm [4, 51], the precalculated dose distribution from a single ray of photons in water is scaled with the density distribution along the ray, disregarding lateral variations. Collapsed cone algorithms [2] also take lateral variations into account and are thus more accurate.

Monte Carlo methods are stochastic methods that explicitly simulate the transport of a large number of particles, successively building up an accurate estimate of the dose distribution. Monte Carlo methods are considered the gold standard for dose calculations in radiotherapy [28, 49]. Their computational complexity is, however, an obstacle to the routine use of Monte Carlo in a clinical setting, although substantial advances have been made by using variance reduction techniques and taking advantage of the graphical processing unit (GPU) to speed up computations [40, 89]. 


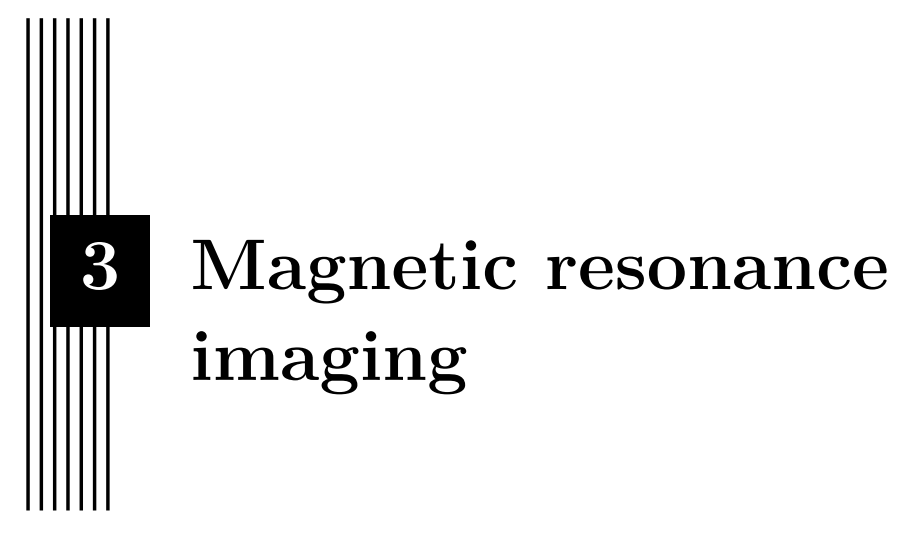

"The imagination of nature is far, far greater than the imagination of man."

Richard P. Feynman (1918-1988)

Since its introduction in the 1970s, magnetic resonance imaging (MRI) has evolved into an indispensable tool for medical imaging. Its excellent soft tissue contrast and inherent patient safety [29] makes MRI preferable to other modalities, such as computed tomography (CT), for a range of imaging tasks. Beyond diagnostic applications, MRI also has a prominent role in radiotherapy planning [79].

Clinical MR scanners create a magnetic field with typical strengths of 1.5 or 3 Tesla (T) - about 50000 times stronger than Earth's magnetic fieldto amplify the effect of a quantum mechanical phenomenon known as nuclear magnetic resonance (NMR). It is based on the fact that protons and neutrons, which make up every atomic nucleus, have an intrinsic quantum property called spin. These spins align either parallel or anti-parallel with an external magnetic field. There is a slight energy difference between the two statescorresponding to the energy of a radio frequency (RF) photon. This implies that radio frequency emitters and receivers can be used to probe the image subject, and measure the strength of the re-emitted signal from different areas. Among biologically relevant elements, hydrogen is, by far, easiest to detect; 
it is the most abundant element and is also relatively sensitive to an external magnetic field.

Since nuclear magnetic resonance is an intrinsically quantum mechanical phenomenon, a thorough description of the signal origin requires a quantum mechanical perspective [1, 15, 36]. Such a description is given in the following section. Readers unfamiliar with quantum mechanics may instead refer to texts providing a classical treatment (which is perhaps more intuitive but can be misleading) [57, 65]. The rest of the chapter then describes how the magnetic resonance phenomena can be used to create images with various contrasts.

\subsection{The origin of the MR signal}

Nuclear magnetic resonance is based on a quantum mechanical property called spin: an intrinsic angular momentum carried by elementary particles - of which the proton is of primary interest. The proton has spin $1 / 2$, which means that measuring, say, the $z$ component of its spin angular momentum $\mathbf{S}$ can return either plus or minus $\frac{1}{2} \hbar$ ('spin up' or 'spin down'), where $\hbar$ is Planck's constant divided by $2 \pi$. The spin is directly tied to the magnetic moment $\boldsymbol{\mu}$ of the particle. For a proton at rest, the relation is given by

$$
\boldsymbol{\mu}=\gamma \mathbf{S}
$$

where $\gamma=42.58 \mathrm{MHz} / \mathrm{T}$ is the gyromagnetic ratio. In general, the gyromagnetic ratio is different for different particles. The existence of a magnetic moment means that the particle will be affected by an external magnetic field. More precisely, the potential energy $E$ of a proton in an external magnetic field $\mathbf{B}=B \hat{\mathbf{z}}$ is

$$
E=-\boldsymbol{\mu} \cdot \mathbf{B}
$$

The corresponding Hamiltonian operator can, in matrix form, be written as

$$
H=-\gamma B S_{z}=\left(\begin{array}{cc}
-\frac{1}{2} \hbar \omega_{0} & 0 \\
0 & \frac{1}{2} \hbar \omega_{0}
\end{array}\right) .
$$

The quantity $\omega_{0}=\gamma B$ is called the Larmor frequency, for a reason soon to be explained. It follows by inspection that the Hamiltonian (3.3) has the eigenstates

$$
\boldsymbol{\psi}_{\uparrow}=\left(\begin{array}{l}
1 \\
0
\end{array}\right), \quad \boldsymbol{\psi}_{\downarrow}=\left(\begin{array}{l}
0 \\
1
\end{array}\right),
$$

with the corresponding eigenvalues

$$
E_{\uparrow}=-\frac{1}{2} h \omega_{0}, \quad E_{\downarrow}=\frac{1}{2} h \omega_{0} .
$$


This shows that the energy is lowest when the magnetic moment is aligned with the external field. This splitting of the energy levels is known as the Zeeman effect.

The time-evolution of the magnetic moment follows from the timedependent Schrödinger equation [35],

$$
i \hbar \frac{\partial \boldsymbol{\psi}}{\partial t}=H \boldsymbol{\psi}
$$

Expanding $\boldsymbol{\psi}(t)$ using the eigenstates (3.4) gives

$$
\boldsymbol{\psi}(t)=c_{\uparrow} \psi_{\uparrow} e^{-i E_{\uparrow} t / \hbar}+c_{\downarrow} \psi_{\downarrow} e^{-i E_{\downarrow} t / \hbar}=\left(\begin{array}{c}
c_{\uparrow} e^{i \omega_{0} t / 2} \\
c_{\downarrow} e^{-i \omega_{0} t / 2}
\end{array}\right),
$$

where the constants $c_{\uparrow}$ and $c_{\downarrow}$ are determined by the initial conditions. From the normalization requirement, it follows that a natural way of rewriting these are as $c_{\uparrow}=\cos (\alpha / 2)$ and $c_{\downarrow}=\sin (\alpha / 2)$ for a fixed angle $\alpha$. Since the magnetic moment $\boldsymbol{\mu}$ is related to $\mathbf{S}$ by a constant, we find that its expected value is

$$
\begin{aligned}
& \left\langle\mu_{x}\right\rangle=\gamma \boldsymbol{\psi}(t)^{\dagger} S_{x} \boldsymbol{\psi}(t)=\frac{\gamma \hbar}{2} \sin \alpha \cos \left(\omega_{0} t\right), \\
& \left\langle\mu_{y}\right\rangle=\gamma \boldsymbol{\psi}(t)^{\dagger} S_{y} \boldsymbol{\psi}(t)=-\frac{\gamma \hbar}{2} \sin \alpha \sin \left(\omega_{0} t\right), \\
& \left\langle\mu_{z}\right\rangle=\gamma \boldsymbol{\psi}(t)^{\dagger} S_{z} \boldsymbol{\psi}(t)=\frac{\gamma \hbar}{2} \cos \alpha
\end{aligned}
$$

As illustrated in figure 3.1, this means that $\langle\boldsymbol{\mu}\rangle$ is tilted at a constant angle $\alpha$ to the direction of the external magnetic field and precesses about it with the Larmor frequency $\omega_{0}$. For a spin 1/2 particle $\alpha= \pm 54.44^{\circ}$ [57].

From equation (3.2), we have that the energy difference between the spin up and spin down states is $\Delta E=\hbar \omega_{0}$. Now-according to the MaxwellBoltzmann theory in statistical mechanics - the probability of finding the system in a state with energy $\epsilon$ when in thermal equilibrium with a reservoir at temperature $T$ (body temperature) is given by

$$
p(\epsilon)=\frac{e^{-\epsilon / k_{B} T}}{Z},
$$

where the partition function $Z$ serves as a normalization factor and $k_{B}$ is Boltzmann's constant. Consequently, there is a larger probability of finding a proton in the low energy state, i.e. with spin parallel to the magnetic field (spin up).

At physiological temperatures and with a magnetic field of $1 \mathrm{~T}$, there are about 3 spins per million more in the low energy state. This might not seem much, but considering that the number of protons in a sample, $N$, is on the order of Avogadro's constant $\left(6.022 \cdot 10^{23}\right)$ it produces an observable magnetic moment. 


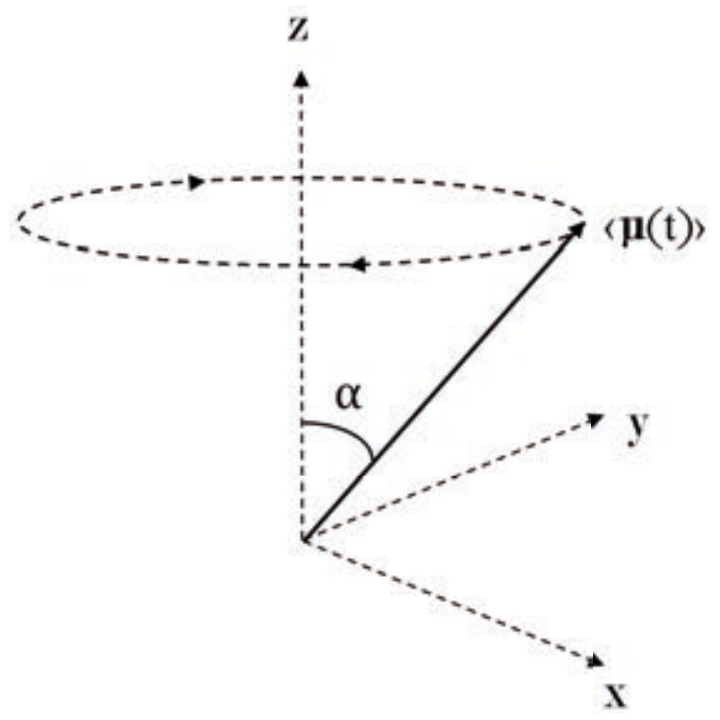

Figure 3.1: The expected value of the magnetic moment $\langle\boldsymbol{\mu}\rangle$ is tilted at a constant angle $\alpha$ to the direction of the external magnetic field and precesses about it with the Larmor frequency $\omega_{0}$.

The excess number of protons in the low energy state produces a net magnetization $\mathbf{M}=N\langle\boldsymbol{\mu}\rangle$. At equilibrium, it points in the direction of the magnetic field, referred to as the longitudinal magnetization $M_{z}$. The component of the magnetization orthogonal to the external magnetic field is called the transverse component; it is often expressed using the complex notation $M_{x y}=M_{x}+i M_{y}$. At equilibrium it is zero, as the transverse components of the magnetic moments are distributed in random directions and their magnetic effects therefore cancel each other.

\subsection{Excitation and relaxation}

In the previous section, we saw how nuclear magnetism can be used to produce a net magnetization in a sample. Creating an actual image requires clever manipulation of the net magnetization. This is where the resonance part of magnetic resonance imaging comes in; only photons with an energy that exactly matches the energy difference between the spin states can influence the spin. Such photons are typically in the radio frequency $(\mathrm{RF})$ range for magnetic fields on the order of $1 \mathrm{~T}$. To see how RF pulses affect the net magnetization, first consider a coordinate system that rotates about the $z$ - 
axis at the Larmor frequency,

$$
\begin{aligned}
& \hat{x}^{\prime}=\hat{x} \cos \left(\omega_{0} t\right)-\hat{y} \sin \left(\omega_{0} t\right), \\
& \hat{y}^{\prime}=\hat{x} \sin \left(\omega_{0} t\right)+\hat{y} \cos \left(\omega_{0} t\right), \\
& \hat{z}^{\prime}=\hat{z} .
\end{aligned}
$$

Assuming that the radio frequency pulse is left circularly polarized and has a time-dependent magnetic field strength $B_{1}(t)$, its associated magnetic field can be written simply as $\mathbf{B}_{1}(t)=B_{1}(t) \hat{x}^{\prime}$. From a derivation similar to the one for the static case, one may show [1, 36] that the expected value of the magnetic moment after applying the pulse for a time $\tau$ are

$$
\begin{aligned}
& \left\langle\mu_{x^{\prime}}(\tau)\right\rangle=\left\langle\mu_{x^{\prime}}(0)\right\rangle, \\
& \left\langle\mu_{y^{\prime}}(\tau)\right\rangle=\left\langle\mu_{y^{\prime}}(0)\right\rangle \cos \theta+\left\langle\mu_{z^{\prime}}(0)\right\rangle \sin \theta, \\
& \left\langle\mu_{z^{\prime}}(\tau)\right\rangle=-\left\langle\mu_{y^{\prime}}(0)\right\rangle \sin \theta+\left\langle\mu_{z^{\prime}}(0)\right\rangle \cos \theta,
\end{aligned}
$$

where we have introduced the flip angle

$$
\theta=\int_{0}^{\tau} \gamma B_{1}(t) d t
$$

Thus, the RF pulse rotates the magnetization about its axis (here $\hat{x}^{\prime}$ ) with an angle given by the flip angle.

When the pulse is turned off the sample will start to relax to its equilibrium state. This happens at different time scales for the transverse and longitudinal magnetization. Longitudinal magnetization, with characteristic time $T_{1}$, is limited by how fast the excited spins release their energy to the surrounding lattice. Transverse relaxation, with characteristic time $T_{2}$, is the gradual loss of precessional coherence, in other words the precessional frequencies of the individual spins spread out over time. Both relaxation processes are usually ascribed to time-dependent microscopic fluctuations in the magnetic field arising from the ever-present thermal motion [1].

The relaxational effects are captured by the phenomenological Bloch equations which, in the rotating frame, can be written as

$$
\begin{aligned}
\frac{d M_{z^{\prime}}}{d t} & =-\frac{M_{z^{\prime}}-M_{z}^{0}}{T_{1}}, \\
\frac{d M_{x^{\prime} y^{\prime}}}{d t} & =-\frac{M_{x^{\prime} y^{\prime}}}{T_{2}},
\end{aligned}
$$

where $M_{\sim}^{0}$ is the longitudinal magnetization at thermal equilibrium. Equation (3.19) yields an exponential regrowth of the longitudinal magnetization, according to

$$
M_{z^{\prime}}(t)=M_{z}^{0}\left(1-e^{-t / T 1}\right)+M_{z^{\prime}}(0) e^{-t / T_{1}}
$$




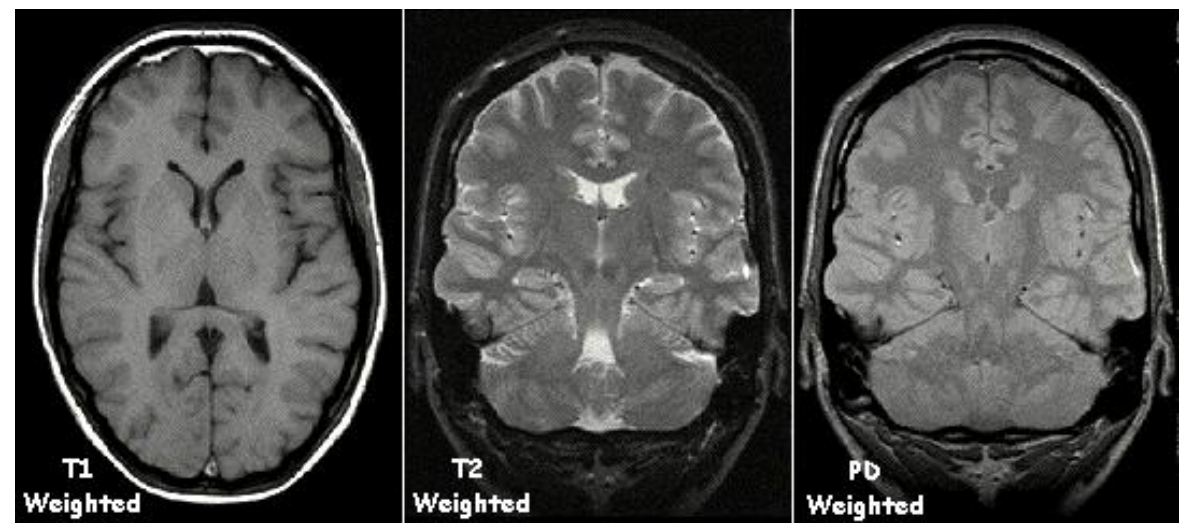

Figure 3.2: Examples of MR images with contrast primarily due to variations in $T_{1}, T_{2}$ or proton density (PD). The left image shows an axial section of a brain whereas the center and right ones show coronal sections.

where $M_{z^{\prime}}(0)$ is the longitudinal magnetization along the $z^{\prime}$-axis immediately after the RF pulse. Similarly, equation (3.20) yields an exponential decay of the transverse magnetization

$$
M_{x^{\prime} y^{\prime}}(t)=M_{x^{\prime} y^{\prime}}(0) e^{-t / T 2},
$$

where $M_{x^{\prime} y^{\prime}}(0)$ is the transverse magnetization immediately after the $R F$ pulse. The precession of the transverse magnetization in the stationary coordinate frame, $M_{x y}=M_{x^{\prime} y^{\prime}} e^{-i \omega_{0} t}$, can be detected by an antenna coil via induction. This is what constitutes the signal in MRI!

The contrast in MR images stems from the tissue dependence of the proton density and the relaxation times $T_{1}$ and $T_{2}$. Scans that primarily achieve contrast from differences in $T_{1}$, called $T_{1}$-weighted scans, achieve a strong signal from fat, gray matter and white matter, whereas the signal from the cerebrospinal fluid $(\mathrm{CSF})$ is weak. $T_{2}$-weighted scans, on the other hand, display a strong response from CSF and intermediate response from fat, gray matter and white matter. It is also possible to acquire proton density (PD) weighted scans, but it is less common. Figure 3.2 illustrates the different appearances of $T_{1^{-}}, T_{2^{-}}$and $\mathrm{PD}$ weighted MR images. Some typical values of the relaxation times are shown in table 3.1. Bone has an extremely short $T_{2}$ relaxation time, $0.4-0.5 \mathrm{~ms}$, which makes it technically difficult - but not impossible - to measure [83, 121, 123]. Because of the short relaxation time, conventional MRI sequences result in a very weak response from bone, thus making it difficult to distinguish from air (which has a low signal because of its low proton density). For applications in radiotherapy, this can be troublesome since bone is the material which attenuates radiation the most and air barely attenuates it at all. In papers II and II, we describe two different approaches 


\begin{tabular}{l|c|c|} 
Tissue type & $T_{1}(\mathrm{~ms})$ & $T_{2}(\mathrm{~ms})$ \\
\hline White matter (WM) & 600 & 80 \\
Gray matter (GM) & 950 & 100 \\
Cerebrospinal fluid (CSF) & 4500 & 2200 \\
Muscle & 900 & 50 \\
Fat & 250 & 60
\end{tabular}

Table 3.1: Typical values of $T_{1}$ and $T_{2}$ for some different tissue types in a magnetic field of strength $1.5 \mathrm{~T}$ [36].

that circumvent this shortcoming of MRI using prior information from CT images.

The presence of inhomogeneities in the external magnetic field can accelerate the transverse relaxation, making its effective characteristic time $T_{2}^{*}$ machine-dependent. The sequence in which RF pulses and magnetic field gradients are applied makes the signal dependent on either $T_{2}$ or $T_{2}^{*}$. This is the subject of section 3.3.

\section{Pulse parameters}

To increase the signal-to-noise ratio (SNR) of MR images, it is common to repeat the same sequence a number of times and average the results. To retain signal strength, however, the longitudinal magnetization must be allowed to recover between repetitions. This places a restriction on the number of times a sequence can be repeated during a given time interval. It is possible to use a repetition time $T_{R}$ such that the longitudinal magnetization only recovers partially between repetitions. After a while the same, steady-state, magnetization will be reached immediately before the next repetition starts. From the Bloch equations, (3.19) and (3.20), it is possible to show that, when using a flip angle $\theta$, the steady-state magnetization is

$$
\begin{aligned}
M_{z^{\prime}}^{s s} & =M_{z}^{0} \frac{\left(1-e^{-T_{R} / T_{1}}\right)}{1-\cos (\theta) e^{-T_{R} / T_{1}}}, \\
M_{x^{\prime} y^{\prime}}^{s s} & =M_{z}^{0} \frac{\left(1-e^{-T_{R} / T_{1}}\right)}{1-\cos (\theta) e^{-T_{R} / T_{1}}} \sin (\theta) e^{-T_{E} / T_{2}} .
\end{aligned}
$$

We see that the repetition time is related to the $T_{1}$ relaxation and that the echo time is related to the $T_{2}$ relaxation.

\subsection{Pulse sequences}

There are three basic contrast mechanisms in MRI: $T_{1}$ relaxation, $T_{2}$ relaxation and proton density (PD). Although it is possible to apply an RF pulse and simply measure the signal as the magnetization returns to equilibrium, 
referred to as Free Induction Decay (FID), this is not what is typically done. As alluded to in the previous section, a clever application of RF pulses and magnetic field gradients makes it possible to accentuate a contrast mechanism of choice.

This section will first describe the gradient system of an MR scanner, which is crucial both for contrast selectivity and, as will be shown later, for spatial encoding of the signal. Then, we will describe two fundamental pulse sequences, the spin echo and the gradient echo, and look at how different pulse parameters result in images with different contrast.

\section{Gradients}

The system generating magnetic field gradients usually consists of three orthogonal gradient coils that generate a time-varying magnetic field $\mathbf{B}(\mathbf{r}, t)=$ $\left(B_{x}, B_{y}, B_{z}\right)$. Because of the much stronger static magnetic field $\mathbf{B}_{0}=B_{0} \hat{z}$, only the part of $\mathbf{B}$ parallel with the $\hat{z}$-axis will make a significant contribution to the magnitude of the magnetic field [15]. Moreover, the gradients are designed to generate a magnetic field that varies linearly with the position $\mathbf{r}$. This means that it is usually sufficient to describe them by their gradients, $\mathbf{G}(t)=\nabla_{\mathbf{r}} B_{z}(\mathbf{r}, t)$.

The usefulness of a spatially varying magnetic field comes from the fact that the Larmor frequency, $\omega=\gamma B$, depends on the local magnitude of the magnetic field. Thus, a spatially varying magnetic field implies a spatially varying frequency

$$
\omega(\mathbf{r})=\gamma\left(B_{0}+\mathbf{r} \cdot \mathbf{G}\right)
$$

Important specifications for a gradient system include the maximum gradient strength and the rate at which the gradient strength can be changed, referred to as the slew rate. Today's clinical scanners have a maximum gradient strength on the order of $50 \mathrm{mT} / \mathrm{m}$ (millitesla per meter) and a slew rate on the order of $100 \mathrm{mT} / \mathrm{m} / \mathrm{s}$. Depending on the application, these specifications may have a strong impact on image quality and/or acquisition time. This was one of the driving factors for the pulse sequence optimization described in papers $\mathrm{III}$ and $\mathrm{IV}$.

\section{Echoes}

Echo sequences usually consist of two equally long parts: a dephasing part and a refocusing part. The two fundamental types of echo sequences, spin echo (SE) and gradient echo (GE), differ in how this dephasing and refocusing is achieved. Both, however, begin with an RF excitation pulse. Often, a spin echo uses a $90^{\circ}$ flip angle whereas a gradient echo uses a smaller flip angle, e.g. $15^{\circ}$. Generally speaking, spin echoes produce higher quality images but gradient echoes enable fast imaging [80]. 


\section{Spin echo}

To create a spin echo, the spins are allowed to dephase naturally after the excitation pulse. After a time $\tau$, a $180^{\circ} \mathrm{RF}$ pulse is applied, reversing the phase angles. An echo is formed when the spins are back in phase again, which happens at the echo time $T_{E}=2 \tau$. This phase reversal trick removes the effects of any magnetic field inhomogeneities, so the echo amplitude will only depend on $T_{2}\left(\operatorname{not} T_{2}^{*}\right)$.

\section{Gradient echo}

To create a gradient echo, a gradient is first applied during a time interval $\tau$ immediately following the excitation pulse. This causes rapid dephasing of the spins. Then the opposite gradient is applied, which rephases the spins. As before, the spins are back in phase again at time $T_{E}=2 \tau$, forming an echo. Contrary to spin echoes, gradient echos do not fully compensate for the inhomogeneity of the magnetic field, so the echo amplitude depends on $T_{2}^{*}$. On the other hand, by decreasing the flip-angle the longitudinal magnetization recovers faster, meaning that the repetition time can be shortened. This, in combination with the absence of a refocusing RF pulse, is why gradient echoes with small flip-angles enable fast imaging.

\subsection{Signal localization}

If we were to apply a pulse sequence as described up to this point, we would get back the combined signal from every spin in the sample - that is not an image! To obtain an image it is necessary to encode where in the sample a signal comes from. This can be achieved with the help of the magnetic field gradients G. Signal localization involves two main steps: selective excitation and spatial encoding.

\section{Selective excitation}

In order to only excite a selected part of the volume, a gradient is applied during the RF pulse. Recall that this creates a spatially varying precession frequency,

$$
\omega(\mathbf{r})=\gamma\left(B_{0}+\mathbf{r} \cdot \mathbf{G}\right) .
$$

Only the protons at spatial locations where the precession frequency matches the RF frequency will be excited.

\section{Spatial encoding}

After a part of the volume has been excited, spatial information can be encoded into a signal during the free precession period. To see how, we first introduce what is called the $\mathbf{k}$-space formalism. A spin located at position $\mathbf{r}$ 
subjected to a time-varying gradient $\mathbf{G}(t)$ will, in the rotating frame, acquire the phase

$$
\phi(\mathbf{r}, t)=\int_{0}^{t} \gamma \mathbf{r} \cdot \mathbf{G}\left(t^{\prime}\right) d t^{\prime}=\mathbf{r} \cdot \gamma \int_{0}^{t} \mathbf{G}\left(t^{\prime}\right) d t^{\prime}=\mathbf{r} \cdot \mathbf{k}(t),
$$

where we have introduced

$$
\mathbf{k}(t)=\gamma \int_{0}^{t} \mathbf{G}\left(t^{\prime}\right) d t^{\prime}
$$

Notationally, however, the time-dependence of $\mathbf{k}$ is often suppressed.

The MRI signal, $S(t)$, is due to the transverse magnetization (in the stationary coordinate frame). It can be detected by an antenna coil thanks to the principle of electromagnetic induction [57]. The signal received thus depends on the contributions from all spins in the object,

$$
\begin{aligned}
S(t) & \propto \int M_{x y}(\mathbf{r}, t) d \mathbf{r}=\int M_{x^{\prime} y^{\prime}}(\mathbf{r}, 0) e^{-t / T_{2}} e^{-i\left(\omega_{0} t+\phi\right)} d \mathbf{r} \\
& =e^{-t / T_{2}} e^{-i \omega_{0} t} \int M_{x^{\prime} y^{\prime}}(\mathbf{r}, 0) e^{-i \mathbf{k} \cdot \mathbf{r}} d \mathbf{r}
\end{aligned}
$$

The phase factor $e^{-i \omega_{0} t}$ is removed during the detection using a demodulation procedure. Then, by denoting the spin density as $\rho(\mathbf{r})=M_{x^{\prime} y^{\prime}}(\mathbf{r}, 0)$, and omitting the factor $e^{-t / T_{2}}$, we may express the signal (3.30) as

$$
S(\mathbf{k}) \propto \int \rho(\mathbf{r}) e^{-i \mathbf{k} \cdot \mathbf{r}} d \mathbf{r} .
$$

This shows that what we measure is basically the Fourier transform of the spin density. To create an image of $\rho(\mathbf{r})$ one therefore has to sample the signal $S(\mathbf{k})$ in $\mathbf{k}$-space. As shown in figure 3.3, this can be done in different ways. The easiest way is to sample on a Cartesian grid, as the image can then be reconstructed using the inverse Fourier transform

$$
\rho(\mathbf{r}) \propto(2 \pi)^{-d / 2} \int S(\mathbf{k}) e^{i \mathbf{k} \cdot \mathbf{r}} d \mathbf{k}
$$

where $d$ is the dimension. The traversal of $\mathbf{k}$-space can be done using frequency encoding and/or phase encoding. Frequency encoding involves sampling the signal at successive intervals in the presence of a read-out gradient. This corresponds to moving along a continuous trajectory in $\mathbf{k}$-space. Phase encoding is achieved by first applying a gradient for a time-interval, during which the spins acquire a phase according to equation (3.27), and then performing a measurement. This corresponds to a jump in $\mathbf{k}$-space. 

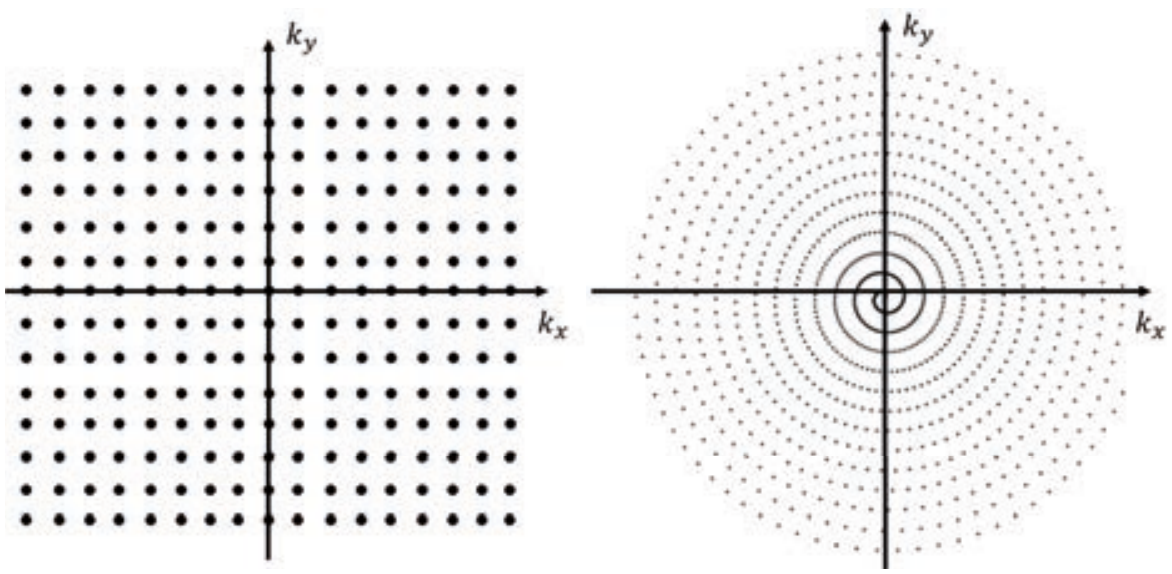

Figure 3.3: Sampling of $\mathbf{k}$-space can be performed in different ways. The left image shows a 2D Cartesian sampling pattern and the right image shows a 2D spiral sampling pattern. Image adapted, with permission, from [24]. 



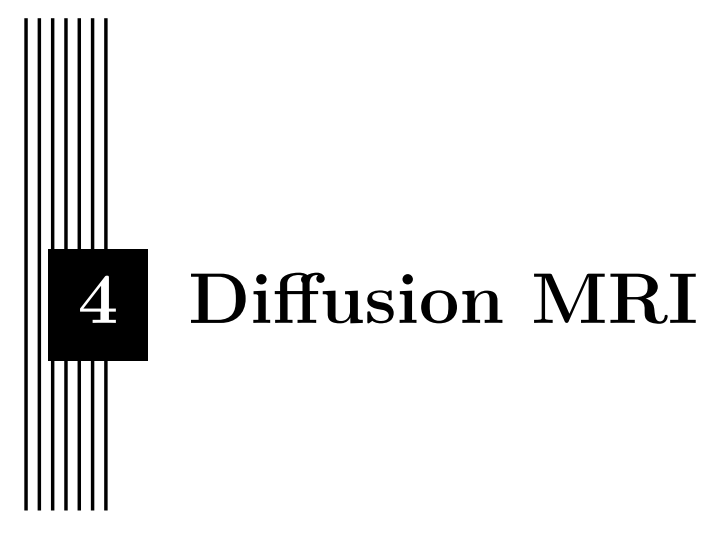

"Mathematics is not a spectator sport"

George M. Phillips (1938-)

Already in 1950, when the idea of spin echoes was first proposed, it was realized that diffusion would affect nuclear magnetic resonance measurements [37]. The reason is that nuclear spins have a phase which is determined by the history of the magnetic field they have experienced. Conversely, by manipulating the applied magnetic field, diffusion MRI (dMRI) serves as a probe of molecular motion. An interesting feature of diffusion MRI is that the scale of what is measured is determined by how far the molecules diffuse during an experiment - not by the resolution in the reconstructed image. In a typical dMRI experiment, the characteristic diffusion length is in the micrometer range, same as the order of cell sizes. This is why diffusion MRI is sometimes referred to as microstructure imaging.

This chapter covers the underlying principles of molecular translational motion and outlines how magnetic resonance can reveal those dynamics.

\subsection{Diffusion}

The internal energy of a substance is stored in the molecular motion of its constituent particles. Under normal circumstances - when the temperature is 
not near absolute zero - a particle chosen at random will almost surely be on the move. A common misconception is that this particle's motion is random. It is not; it follows the laws of mechanics. On the other hand, the particle density in a liquid substance makes molecular collisions inevitable. So, if you sample the particle's position at a time scale much larger than the time scale of molecular collisions, you can effectively treat the motion as random. The particle motion is thus appropriately described as a stochastic process.

\section{Stochastic processes}

As we have seen above, the displacement of a particle from one instance in time to another can be considered a random variable. As time evolves, the particle will perform a sequence of random displacements. This is conveniently described using the concept of a stochastic process [116], which is the random function obtained in the limit of an infinite number of time steps. The mean of a stochastic process $x(t)$ is an average over the (possibly infinite) number of possible realizations, weighted by their corresponding probabilities

$$
\mu_{x}(t)=\langle x(t)\rangle=\int x p(x, t) d x .
$$

In general, the mean of a stochastic process is a function. However, many stochastic processes of interest are stationary, which means that their statistical properties, including the mean, do not change over time. So, the mean of a stationary process is a constant that can be subtracted. It is therefore customary to assume that the mean is zero for a stationary process.

In addition to the mean, the (auto)covariance function is often used to characterize a stochastic process. Given times $t$ and $t_{0}$, it is defined as

$$
\begin{aligned}
k_{x}\left(t, t_{0}\right) & =\left\langle\left(x(t)-\mu_{x}(t)\right)\left(x\left(t_{0}\right)-\mu_{x}\left(t_{0}\right)\right)\right\rangle \\
& =\left\langle x(t) x\left(t_{0}\right)\right\rangle-\mu_{x}(t) \mu_{x}\left(t_{0}\right) .
\end{aligned}
$$

For a stationary process, the covariance function only depends on the time difference $\tau=\left|t-t_{0}\right|$. Hence, assuming zero mean, the covariance function simplifies to

$$
k_{x}(\tau)=\langle x(\tau) x(0)\rangle
$$

\section{The Langevin equation}

A powerful way of modeling diffusion is to represent the effect of molecular collisions by a fluctuating force $\xi(t)$, that is characterized as white noise

$$
\begin{aligned}
\mu_{\xi}(t) & =0, \\
k_{\xi}\left(t, t_{0}\right) & =\delta\left(t-t_{0}\right) .
\end{aligned}
$$


If we also allow for an external force $\mathbf{f}(\mathbf{r})$, then Newton's equation of motion for a particle of mass $m$ in a viscous medium, with constant friction coefficient $\zeta$, subject to a fluctuating force is

$$
m \ddot{\mathbf{r}}=\mathbf{f}(\mathbf{r})-\zeta \dot{\mathbf{r}}+\sigma \boldsymbol{\xi}
$$

where the constant $\sigma$ corresponds to the strength of the fluctuating force. This is known as the Langevin equation [18, 54, 116]. In the strong friction limit, which applies to diffusion MRI [15, 85], $|\zeta \dot{\mathbf{r}}| \gg m \ddot{\mathbf{r}}$. One may thus consider the overdamped Langevin equation instead:

$$
\zeta \dot{\mathbf{r}}=\mathbf{f}(\mathbf{r})+\sigma \boldsymbol{\xi}
$$

Instead of considering individual particles, it is also possible to consider their collective behavior, in a statistical sense. Of particular interest is the probability that a particle at position $\mathbf{r}_{0}$ at time $t_{0}$ moves to the position $\mathbf{r}$ at a later time $t$. This can be expressed as a conditional probability distribution $p\left(\mathbf{r}, t \mid \mathbf{r}_{0}, t_{0}\right)$, and is often referred to as the propagator (or Green's function). The Fokker-Planck equation [82, 116] links the description of a particle's position as a stochastic differential equation to a partial differential equation for the propagator. In particular, the Fokker-Planck equation corresponding to equation (4.7) is [16, 82]

$$
\frac{\partial}{\partial t} p\left(\mathbf{r}, t \mid \mathbf{r}_{0}, t_{0}\right)=\left(D \nabla^{2}-\nabla \cdot \frac{\mathbf{f}(\mathbf{r})}{\zeta}\right) p\left(\mathbf{r}, t \mid \mathbf{r}_{0}, t_{0}\right),
$$

where we have introduced $D=\sigma^{2} /\left(2 \zeta^{2}\right)$, which is known as the diffusivity. In the case of free diffusion in a homogeneous medium, $D$ is constant and $\mathbf{f}(\mathbf{r})=0$. Then, equation (4.8) reduces to

$$
\frac{\partial}{\partial t} p\left(\mathbf{r}, t \mid \mathbf{r}_{0}, t_{0}\right)=D \nabla^{2} p\left(\mathbf{r}, t \mid \mathbf{r}_{0}, t_{0}\right)
$$

Given the initial position of a particle, $p\left(\mathbf{r}, t \mid \mathbf{r}, t_{0}\right)=\delta\left(\mathbf{r}-\mathbf{r}_{0}\right)$ and requiring that the probability vanishes at infinity, the solution to equation (4.9) is

$$
p\left(\mathbf{r}, t \mid \mathbf{r}_{0}, t_{0}\right)=(4 \pi D \tau)^{-3 / 2} \exp \left(-\frac{\left(\mathbf{r}-\mathbf{r}_{0}\right)^{2}}{4 D \tau}\right),
$$

where, again, $\tau=t-t_{0}$.

By considering a small displacement of a particle in a system at equilibrium, Einstein was able to show [15, 23] that $D=k_{B} T / \zeta$, where $k_{B}$ is Boltzmann's constant and $T$ is the temperature. Einstein's result is an example of a fluctuation-dissipation relation [52, 62]. 


\section{Diffusivity}

From the Gaussian nature of the propagator (4.10) it follows immediately that, in a homogeneous medium, $\left\langle\left(\mathbf{r}(t)-\mathbf{r}\left(t_{0}\right)\right)^{2}\right\rangle=6 D t$, and similarly in one dimension $\left\langle\left(x(t)-x\left(t_{0}\right)\right)^{2}\right\rangle=2 D t$. This can be used to define the diffusivity $D$ as follows,

$$
D=\lim _{t \rightarrow \infty} \frac{\left\langle\left(x(t)-x\left(t_{0}\right)\right)^{2}\right\rangle}{2 t} .
$$

\section{The diffusion tensor}

The definition of the diffusivity (4.11) is straightforward to generalize to the case of anisotropic diffusion, i.e. when the diffusivity is direction dependent. This is done by having $D$ also refer to the diffusion tensor

$$
D=\left(\begin{array}{lll}
D_{x x} & D_{x y} & D_{x z} \\
D_{y x} & D_{y y} & D_{y z} \\
D_{z x} & D_{z y} & D_{z z}
\end{array}\right)
$$

with elements defined as

$$
D_{\alpha \beta}=\lim _{t \rightarrow \infty} \frac{\left\langle\left(x_{\alpha}(t)-x_{\alpha}\left(t_{0}\right)\right)\left(x_{\beta}(t)-x_{\beta}\left(t_{0}\right)\right)\right\rangle}{2 t}, \quad \alpha, \beta \in\{x, y, z\} .
$$

Clearly, $D$ is always symmetric. Whether $D$ refers to a scalar or a tensor should be clear from the context. The observation that diffusion is anisotropic in nervous tissue has been one of the main reasons for the interest in diffusion MRI [8, 9, 42, 55].

\section{Path integration}

We will now derive the probability of a particle following a particular trajectory using what physicists refer to as path integration. The aim is only to convey the general idea - a rigorous derivation requires a fair amount of theory on Wiener processes. Hence, we will derive the one-dimensional result, starting from the equivalent of equation (4.8)

$$
\frac{\partial}{\partial t} p\left(x, t \mid x_{0}, t_{0}\right)=\left(-\frac{\partial}{\partial x} f(x)+D \frac{\partial^{2}}{\partial x^{2}}\right) p\left(x, t \mid x_{0}, t_{0}\right),
$$

where we have absorbed $\zeta$ in the definition of $f(x)$.

For a short time-step $\Delta t$, such that $t=t_{0}+\Delta t$, we may write

$$
\begin{aligned}
p\left(x, t_{0}+\Delta t \mid x_{0}, t_{0}\right) & =p\left(x_{0}, t_{0} \mid x_{0}, t_{0}\right)+\Delta t \frac{\partial}{\partial t} p\left(x_{0}, t_{0}+\Delta t \mid x_{0}, t_{0}\right)+\mathcal{O}\left((\Delta t)^{2}\right) \\
p\left(x, t_{0} \mid x_{0}, t_{0}\right) & =\delta\left(x-x_{0}\right)
\end{aligned}
$$


Combining these expressions with (4.14) we find that, to order $(\Delta t)^{2}$,

$$
\begin{aligned}
p\left(x, t_{0}+\Delta t \mid x_{0}, t_{0}\right) & =\left(1-\frac{\partial}{\partial x} f\left(x_{0}\right) \Delta t+D \frac{\partial^{2}}{\partial x^{2}} \Delta t\right) \delta\left(x-x_{0}\right) \\
& =\left(1-\frac{\partial}{\partial x} f\left(x_{0}\right) \Delta t+D \frac{\partial^{2}}{\partial x^{2}} \Delta t\right) \int_{-\infty}^{\infty} e^{i \omega\left(x-x_{0}\right)} d \omega \\
& =\int_{-\infty}^{\infty}\left(1-i \omega f\left(x_{0}\right) \Delta t-D \omega^{2} \Delta t\right) e^{i \omega\left(x-x_{0}\right)} d \omega \\
& =\int_{-\infty}^{\infty} e^{-i \omega f\left(x_{0}\right) \Delta t-D \omega^{2} \Delta t} e^{i \omega\left(x-x_{0}\right)} d \omega \\
& =\frac{1}{\sqrt{4 \pi D \Delta t}} \exp \left(-\frac{\left.\left(x-x_{0}-f\left(x_{0}\right) \Delta t\right)^{2}\right)}{4 D \Delta t}\right)
\end{aligned}
$$

where, in equation (4.18), we replaced $\delta\left(x-x_{0}\right)$ with its Fourier representation and, in equation (4.20), we used the linearization of the exponential function (which is accurate to second order).

To find the probability for a larger time-step $\left(t-t_{0}\right)$, we subdivide it into a large number $N$ of tiny steps, $\Delta t=\left(t-t_{0}\right) / N$, such that equation (4.21) can be applied for each,

$$
\begin{aligned}
p(x, t) & =\int d x_{N-1} p\left(x, t \mid x_{N-1}, t_{N-1}\right) \ldots \int d x_{0} p\left(x_{1}, t_{1} \mid x_{0}, t_{0}\right) p\left(x_{0}, t_{0}\right) \\
& =\prod_{n=0}^{N-1} \int d x_{n} p\left(x_{n+1}, t_{n+1} \mid x_{n}, t_{n}\right) p\left(x_{0}, t_{0}\right) \\
& \propto \prod_{n=0}^{N-1} \int d x_{n} \exp \left(-\frac{\left.\left(x_{n+1}-x_{n}-f\left(x_{n}\right) \Delta t\right)^{2}\right)}{4 D \Delta t}\right) p\left(x_{0}, t_{0}\right) \\
& \propto \int\left(\prod_{n=0}^{N-1} d x_{n}\right) \exp \left(-\sum_{n=0}^{N-1} \frac{\left.\left(x_{n+1}-x_{n}-f\left(x_{n}\right) \Delta t\right)^{2}\right)}{4 D \Delta t}\right) p\left(x_{0}, t_{0}\right) .
\end{aligned}
$$

In the limit $N \rightarrow \infty$, it is tempting to replace the sum in the exponent by an integral. Alas, stochastic calculus obeys a somewhat different set of rules than ordinary calculus - in particular, the Riemann integral is not valid. Without going into details [126], the expression for the limit of (4.25) is, in Stratonovich sense,

$$
p(x, t) \propto \int \mathcal{D}[x] \exp \left(-\int_{t_{0}}^{t}\left[\frac{(\dot{x}-f(x))^{2}}{4 D}-\frac{1}{2} \frac{d}{d x} f(x)\right] d t^{\prime}\right) p\left(x_{0}, t_{0}\right) .
$$

The generalization to higher dimensions is straightforward, but unwieldy [82].

\subsection{Measuring diffusion with MRI}

In section 3.4 of the previous chapter, we saw that the addition of a spatially varying magnetic field $\mathbf{G}$ made the precession frequency vary spatially, 
according to

$$
\omega(\mathbf{r})=\gamma\left(B_{0}+\mathbf{r} \cdot \mathbf{G}\right) .
$$

Furthermore, we stated that, over time, a spin at position $\mathbf{r}$ would acquire a phase shift with respect to the rotating frame, given by

$$
\phi(\mathbf{r}, t)=\int_{0}^{t} \gamma \mathbf{r} \cdot \mathbf{G}\left(t^{\prime}\right) d t^{\prime}=\mathbf{r} \cdot \gamma \int_{0}^{t} \mathbf{G}\left(t^{\prime}\right) d t^{\prime} .
$$

We will now revise that statement, because what has tacitly been assumed is that the spin is stationary and - given what we have learned about diffusionwe know better! To be clear about this distinction we will, in accordance with [15], henceforth use $\mathbf{g}(t)$, instead of $\mathbf{G}(\mathrm{t})$, to denote a time-varying gradient that is used to encode for motion. Moreover, we will include any spin reversals due to RF pulses through a sign change in $\mathbf{g}(t)$, so that it represents the effective gradient experienced by the spins. The diffusion measurements we will consider typically involve some type of echo (cf. section 3.3), which means that at the echo time, $T_{E}$, we have that $\int_{0}^{T_{E}} g\left(t^{\prime}\right) d t^{\prime}=0$.

To account for diffusion in the expression for the measured signal (3.30), it is convenient to first introduce the local magnetization $m(\mathbf{r}, t)=$ $M_{x^{\prime} y^{\prime}}(\mathbf{r}, t) e^{t / T_{2}}$. In words, the local magnetization is the transverse magnetization in the rotating frame disregarding $T_{2}$-decay. We can then write the measured signal as

$$
S(t) \propto e^{-t / T_{2}} e^{-i \omega_{0} t} \int m(\mathbf{r}, t) d \mathbf{r},
$$

which looks deceptively like before. The interesting part, however, is that because of diffusion, the local magnetization is

$$
\begin{aligned}
m(\mathbf{r}, t) & =\rho(\mathbf{r})\left\langle\exp \left(-i \gamma \int_{t_{0}}^{t} \mathbf{g}\left(t^{\prime}\right) \cdot \mathbf{r}\left(t^{\prime}\right) d t^{\prime}\right)\right\rangle \\
& =\rho(\mathbf{r})\left\langle e^{-i \phi(\mathbf{r}, t)}\right\rangle,
\end{aligned}
$$

where the expectation is over the infinite number of possible trajectories of a diffusing particle ending at $\mathbf{r}$ at time $t$. This equation is fundamental to diffusion MRI and we will have reason to return to it on multiple occasions. Often, we are only interested in the effects of the applied gradient, which can be isolated by normalizing the signal to the non-diffusion weighted case, giving

$$
\begin{aligned}
E(t) & =\int \frac{m(\mathbf{r}, t)}{\rho(\mathbf{r})} d \mathbf{r} \\
& =\int\left\langle e^{-i \phi(\mathbf{r}, t)}\right\rangle d \mathbf{r} .
\end{aligned}
$$




\section{The Bloch-Torrey equation}

As we saw before, an expectation over trajectories, such as in equation (4.31) can be calculated using path integration. The phase shift then enters as another term in the exponent of (4.26). There are deep connections between probability theory and partial differential equations [47, 48]. Among them, the Feynman-Kac formula [46] links expectations over Wiener processes to partial differential equations. Notably, using the Feynman-Kac formula and equation (4.8), it can be shown that [67, 130] the local magnetization is the solution of

$$
\frac{\partial m}{\partial t}=\left(D \nabla^{2}-\nabla \cdot \frac{\mathbf{f}(\mathbf{r})}{\zeta}\right) m-i \gamma \mathbf{g} \cdot \mathbf{r} m,
$$

with the initial condition $m\left(\mathbf{r}_{0}, t_{0}\right)=\rho\left(\mathbf{r}_{0}\right)$. Setting $\mathbf{f}(\mathbf{r})=0$ reduces this equation to the celebrated Bloch-Torrey equation [114], originally derived using hydrodynamics arguments,

$$
\frac{\partial m}{\partial t}=\left(D \nabla^{2}-i \gamma \mathbf{g} \cdot \mathbf{r}\right) m
$$

The expression within parentheses, sometimes referred to as the Bloch-Torrey operator [39], is not Hermitian. Consequently, the local magnetization is not conserved (rather, it decays).

Curiously, setting $D=i /(2 \mu)$ and $m(\mathbf{r}, t)=\psi(\mathbf{r}, t)$ turns the Bloch-Torrey equation into

$$
i \frac{\partial}{\partial t} \psi=-\frac{1}{2 \mu} \nabla^{2} \psi+\gamma \mathbf{g} \cdot \mathbf{r} \psi,
$$

which is nothing but the Schrödinger equation (in units where $\hbar=1$ ) for a particle with the imaginary mass $\mu=i D / 2$ in a potential $V(\mathbf{r}, t)=\gamma \mathbf{g} \cdot \mathbf{r}$ [107]. Strange as they may seem, particles with imaginary mass, referred to as tachyons [26], are fairly well-studied in theoretical physics [67, 88]. It is conceivable that this connection to quantum physics could bring new insights to diffusion MRI.

\section{Solution in the case of free and homogeneous diffusion}

In a few cases, the Bloch-Torrey equation can be solved analytically; the simplest is free diffusion. For ease of presentation, we consider the 1D case:

$$
\frac{\partial m}{\partial t}=\left(D \frac{\partial^{2}}{\partial x^{2}}-i \gamma g(t) x\right) m
$$

Taking the spatial Fourier transform, $\hat{m}(q, t)=\int m(x, t) e^{-i q x} d x$, yields,

$$
\frac{\partial \hat{m}}{\partial t}=-D q^{2} \hat{m}+\gamma g(t) \frac{\partial \hat{m}}{\partial q} .
$$


Since this is a first order PDE, we may use the method of characteristics [20] to transform it into the system of ordinary differential equations

$$
\begin{aligned}
\frac{d t}{d s} & =1, \\
\frac{d q}{d s} & =-\gamma g(t), \\
\frac{d \hat{m}}{d s} & =-D q^{2} \hat{m} .
\end{aligned}
$$

We fix $t(0)=0$, so that equation (4.39) gives $t=s$. Since $g(t)=0$ for $t \leq 0$, it follows from equation (4.40) that

$$
q(t)=-\gamma \int_{0}^{t} g\left(t^{\prime}\right) d t^{\prime}
$$

From equation (4.41) we find that

$$
\hat{m}(s)=\hat{m}(0) \exp \left(-D \int_{0}^{s} q\left(s^{\prime}\right)^{2} d s^{\prime}\right)
$$

Finally, we take the inverse Fourier transform, restricted to the characteristic, and use the initial condition $m(x, 0)=\rho(x)$, to find the sought solution to the Bloch-Torrey equation:

$$
m(x, t)=\rho(x) \exp \left(-D \int_{0}^{t} q\left(t^{\prime}\right)^{2} d t^{\prime}\right) \exp (i q(t) x) .
$$

Following an identical line of reasoning, one may show that the multidimensional generalization is

$$
m(\mathbf{r}, t)=\rho(\mathbf{r}) \exp \left(-\int_{0}^{t} \mathbf{q}\left(t^{\prime}\right)^{T} D \mathbf{q}\left(t^{\prime}\right) d t^{\prime}\right) \exp (i \mathbf{q}(t) \cdot \mathbf{r}) .
$$

Note that at the echo center $\mathbf{q}\left(T_{E}\right)=0$, so the complex exponential disappears. The normalized echo amplitude is thus

$$
E\left(T_{E}\right)=\exp \left(-\int_{0}^{T_{E}} \mathbf{q}\left(t^{\prime}\right)^{T} D \mathbf{q}\left(t^{\prime}\right) d t^{\prime}\right) .
$$

By introducing the measurement tensor (b-tensor) [15, 122]

$$
B=\int_{0}^{T_{E}} \mathbf{q}(t) \mathbf{q}(t)^{T} d t
$$

it is possible to rewrite equation (4.46) in the, perhaps, more expressive form

$$
E\left(T_{E}\right)=e^{-\operatorname{Tr}(B D)} .
$$

Paper III describes a method of numerically optimizing the gradient waveform $\mathbf{g}(t)$ (or, equivalently, $\mathbf{q}(t)$ ) to achieve a b-tensor with maximum diffusion weighting subject to constraints imposed by the MR scanner. This is then used in paper IV to perform a certain type of diffusion measurement called diffusional variance decomposition (DIVIDE), see section 4.4. 


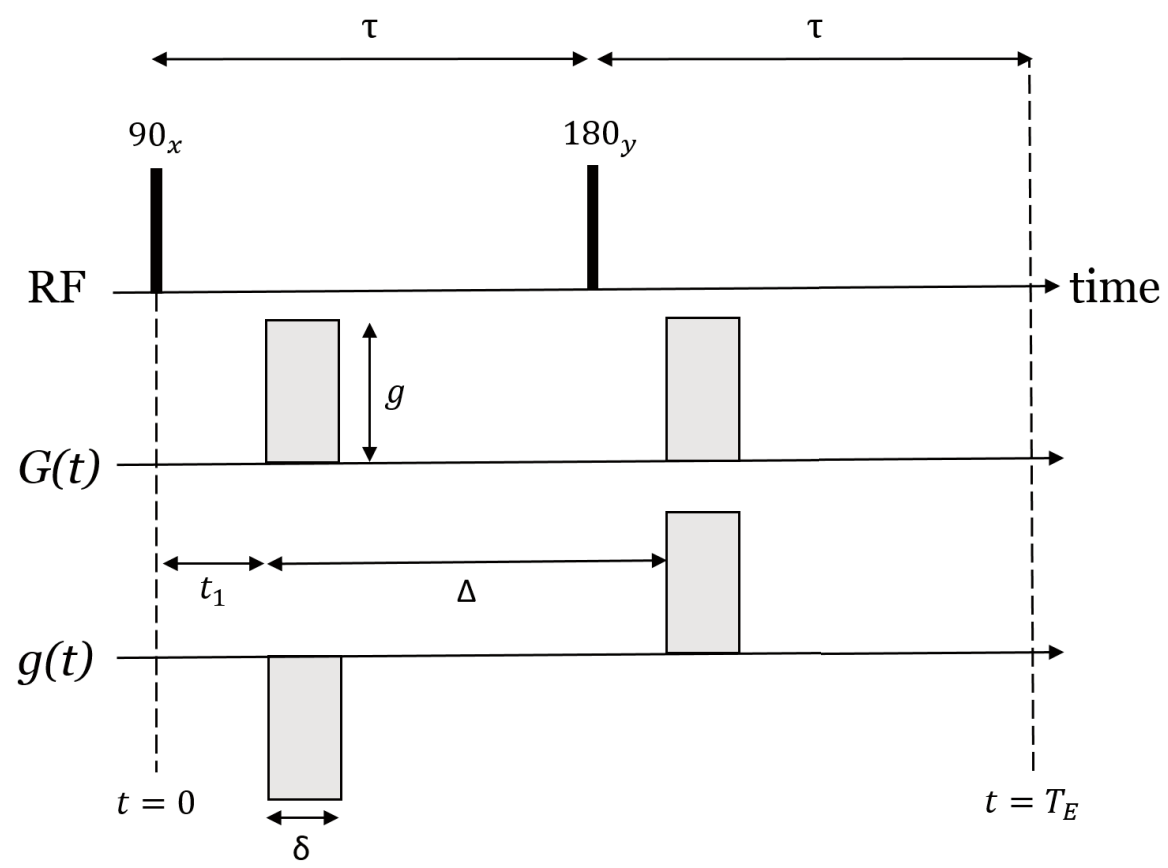

Figure 4.1: The Stejskal-Tanner sequence - the mainstay of diffusion MRI. The top line shows the RF excitations, the middle line the actual gradient sequence applied and the bottom line the effective gradient sequence (with sign change at $180^{\circ}$ pulse).

\subsection{The Stejskal-Tanner experiment}

In 1965, Stejskal and Tanner demonstrated [106] a pulse sequence that still remains the predominant diffusion measurement using magnetic resonance. An example of a Stejskal-Tanner sequence using a spin echo is shown in figure 4.1. Although details in the implementation may vary, the main characteristic of such a measurement it that the effective gradient sequence consists of two blocks of equal magnitudes but opposite directions. The gradient sequence can thus be written

$$
\mathbf{g}(t)= \begin{cases}-\mathrm{g} & t_{1} \leq t \leq t_{1}+\delta \\ \mathbf{g} & t_{1}+\Delta \leq t \leq t_{1}+\Delta+\delta . \\ 0 & \text { otherwise }\end{cases}
$$

Inserting this into (4.46), we find that the signal at the echo time is given by

$$
E\left(T_{E}\right)=\exp \left(-\gamma^{2} \delta^{2}(\Delta-\delta / 3) \mathbf{g}^{T} D \mathbf{g}\right)
$$


For this reason, the echo attenuation (diffusion weighting) is often expressed using the $b$-value, defined as

$$
b=\gamma^{2}\|\mathbf{g}\|^{2} \delta^{2}(\Delta-\delta / 3) .
$$

Obviously, this definition presupposes that a Stejskal-Tanner sequence is used.

By repeating such an experiment with different gradient directions, it is possible to estimate the full diffusion tensor. This is referred to as diffusion tensor imaging (DTI) [8]. The diffusion tensor can be reduced to various scalar indices. The perhaps most widespread are the mean diffusivity (MD) and fractional anisotropy (FA),

$$
\begin{aligned}
& M D=\frac{\operatorname{Tr}(D)}{3}, \\
& F A=\sqrt{\frac{1}{2}\left(3-\frac{\operatorname{Tr}(D)^{2}}{\operatorname{Tr}\left(D^{2}\right)}\right)} .
\end{aligned}
$$

There are many equivalent ways of expressing FA, but the one above elucidates its nonlinear dependence on $D[71]$.

\section{The narrow pulse approximation}

When the diffusivity is not constant it is in most cases impossible to obtain a closed form solution to the Bloch-Torrey equation. An important exception is for a Stejskal-Tanner type experiment where the gradient duration $\delta$ is so short that motion during its application can be neglected. This is referred to as the narrow pulse approximation. Mathematically, it implies that the gradient sequence can be approximated as the sum of two Dirac pulses

$$
\mathbf{g}(t)=\mathbf{q}\left[\delta\left(t-\left(t_{1}+\Delta\right)\right)-\delta\left(t-t_{1}\right)\right]
$$

where, in this expression, $\mathbf{q}=\gamma \delta \mathbf{g}$ is a constant. This notation may be confusing (since it is inconsistent with equation (4.42)) but the reason we use it anyway is that imaging under the narrow pulse approximation has an established name: q-space imaging. To simplify, we let $\mathbf{r}_{1}=\mathbf{r}\left(t_{1}\right), \mathbf{r}_{2}=\mathbf{r}\left(t_{1}+\Delta\right)$ and $\mathbf{R}=\mathbf{r}_{2}-\mathbf{r}_{1}$. Further we define the ensemble averaged propagator (EAP),

$$
P(\mathbf{R}, \Delta)=\int p\left(\mathbf{r}_{1}+\mathbf{R} \mid \mathbf{r}_{1}\right) p\left(\mathbf{r}_{1}\right) d \mathbf{r}_{1} .
$$

Referring to equation (4.33), the normalized echo amplitude effectively reduces to an average over the phase differences at the two sample times $t=t_{1}$ and 
$t=t_{1}+\Delta$

$$
\begin{aligned}
E(\mathbf{q}, \Delta) & =\int\left\langle e^{-i \mathbf{q} \cdot\left(\mathbf{r}_{2}-\mathbf{r}_{1}\right)}\right\rangle d \mathbf{r}_{2} \\
& =\iint p\left(\mathbf{r}_{2} \mid \mathbf{r}_{1}\right) p\left(\mathbf{r}_{1}\right) e^{-i \mathbf{q} \cdot\left(\mathbf{r}_{2}-\mathbf{r}_{1}\right)} d \mathbf{r}_{1} d \mathbf{r}_{2} \\
& =\int\left(\int p\left(\mathbf{r}_{1}+\mathbf{R} \mid \mathbf{r}_{1}\right) p\left(\mathbf{r}_{1}\right) d \mathbf{r}_{1}\right) e^{-i \mathbf{q} \cdot \mathbf{R}} d \mathbf{R} \\
& =\int P(\mathbf{R}, \boldsymbol{\Delta}) e^{-i \mathbf{q} \cdot \mathbf{R}} d \mathbf{R} .
\end{aligned}
$$

In other words, the echo amplitude in a q-space imaging experiment is nothing but the Fourier transform of the EAP (4.55). This holds true without any assumptions on the underlying diffusivity. Consequently, it is possible to estimate the EAP by performing measurements on a dense Cartesian grid in qspace and performing an inverse Fourier transform. However, this procedure, referred to as diffusion spectrum imaging [120], requires such a large number of q-space samples that the acquisition time becomes too long for routine use. In paper $\mathrm{V}$, we describe a machine learning method that makes it possible to estimate the q-space signal based on far fewer samples than required by diffusion spectrum imaging. In particular, we show how to resample data acquired on multiple shells in q-space onto a Cartesian grid. As an alternative to the inverse Fourier transform, we also describe a theoretically well-founded reconstruction method that respects the probabilistic nature of the EAP. We evaluate our method, in part, using the estimation error of a scalar index called the return-to-origin probability (RTOP). It is defined as

$$
R T O P=P(0, \Delta)=\int E(\mathbf{q}, \Delta) d \mathbf{q}
$$

\section{The apparent diffusion coefficient}

Most of the results derived up to this point have assumed that the diffusing particles are free to move without surfaces or boundaries restricting them. In such cases we expect that the average propagator is a Gaussian, as shown in the previous section. When diffusion is restricted, this is no longer true. Of course it is still possible to fit the echo amplitude with an expression of the form $e^{-b D_{\mathrm{app}}}$, and many people do, but the precise meaning of the apparent diffusion coefficient $D_{\text {app }}$ (also known as ADC) is in most cases unclear [34]. The apparent diffusion coefficient is not an intrinsic property of the medium, but depends on the details of the experiment. Thus, reporting the value of $D_{\text {app }}$ without an accurate description of the experimental procedure is, strictly speaking, nonsensical. Nevertheless, use - and often misuse of the apparent diffusion coefficient is widespread in the clinical MRI literature [34, 118. 


\subsection{Diffusional variance decomposition (DIVIDE)}

Diffusional variance decomposition (DIVIDE) [110] is a technique used in paper IV to separate those effects that are due to variations in diffusivity and those that are due to variations in orientation and shape. The underlying assumption is that a voxel can be modeled as an ensemble of microenvironments, each described by a diffusion tensor [122]. In this context, the diffusion tensor $D$ is considered a stochastic variable and, using equation (4.48), the normalized signal is given by

$$
E(B)=\int e^{-\operatorname{Tr}(B D)} p(D) d D=\left\langle e^{-\operatorname{Tr}(B D)}\right\rangle .
$$

The last equality shows that, up to a minus sign, this is the moment generating function of $p(D)$.

We will now describe a simple model that actually generalizes the theory hitherto used for DIVIDE. Suppose that the effective diffusion tensor for a voxel is given by

$$
D=\sum_{i=1}^{n} x_{i} x_{i}^{T}, \quad x_{i} \sim \mathcal{N}(0, V), \quad i=1, \ldots, n .
$$

This implies that $p(D)$ follows the Wishart distribution [127], which is the multivariate generalization of the gamma distribution. The Wishart distribution is widely used in multivariate statistics [75], and has previously been used as an extension to DTI in Stejskal-Tanner type experiments [44]. In the general case $x_{i} \in \mathbb{R}^{p \times 1}$,

$$
p(D \mid V, n)=\frac{1}{|V|^{\frac{n}{2}}} \frac{|D|^{(n-p-1) / 2}}{2^{\frac{n p}{2}} \Gamma_{p}\left(\frac{n}{2}\right)} e^{-\frac{1}{2} \operatorname{Tr}\left(V^{-1} D\right)},
$$

where $\Gamma_{p}$ is the multivariate gamma function. Inserting this in equation (4.61) yields

$$
\begin{aligned}
E(B) & =\int e^{-\operatorname{Tr}(B D)} \frac{1}{|V|^{\frac{n}{2}}} \frac{|D|^{(n-p-1) / 2}}{2^{\frac{n p}{2}} \Gamma_{p}\left(\frac{n}{2}\right)} e^{-\frac{1}{2} \operatorname{Tr}\left(V^{-1} D\right)} d D \\
& =\frac{\left|\frac{1}{2} B^{-1}+V\right|^{\frac{n}{2}}}{|V|^{\frac{n}{2}}} \underbrace{\int p\left(D \mid \frac{1}{2} B^{-1}+V, n\right) d D}_{=1} \\
& =|I+2 B V|^{-\frac{n}{2}} .
\end{aligned}
$$

The mean and covariance of the Wishart distribution are

$$
\begin{aligned}
\langle D\rangle & =n V, \\
\operatorname{Var}[\operatorname{vec}(D)] & =n\left(I+K_{p p}\right)(V \otimes V),
\end{aligned}
$$


where $K_{p p}$ is the commutation matrix [61]. Equation 4.66 can thus be expressed as

$$
E(B)=\left|I+\frac{B\langle D\rangle}{(n / 2)}\right|^{-\frac{n}{2}} .
$$

In DIVIDE, one averages the signal over all orientations ("powder average"), which means that it is the one-dimensional apparent diffusion coefficient that is considered. In that case, the equations (4.68) and (4.69) can be written as

$$
\begin{aligned}
\operatorname{Var}[D] & =2 n V^{2}, \\
E(B) & =\left(1+b \cdot \frac{\operatorname{Var}[D]}{\langle D\rangle}\right)^{-\frac{\langle D\rangle^{2}}{\operatorname{Var}[D]}},
\end{aligned}
$$

where the latter is the one used in paper IV.

As already demonstrated by DIVIDE, diffusion encoding with continuous waveforms provide new ways of probing tissue microstructure. And yet - as shown above - it is but a special case of a more general methodology. I note with excitement that we may well be on the verge of a paradigm shift in diffusion MRI. 



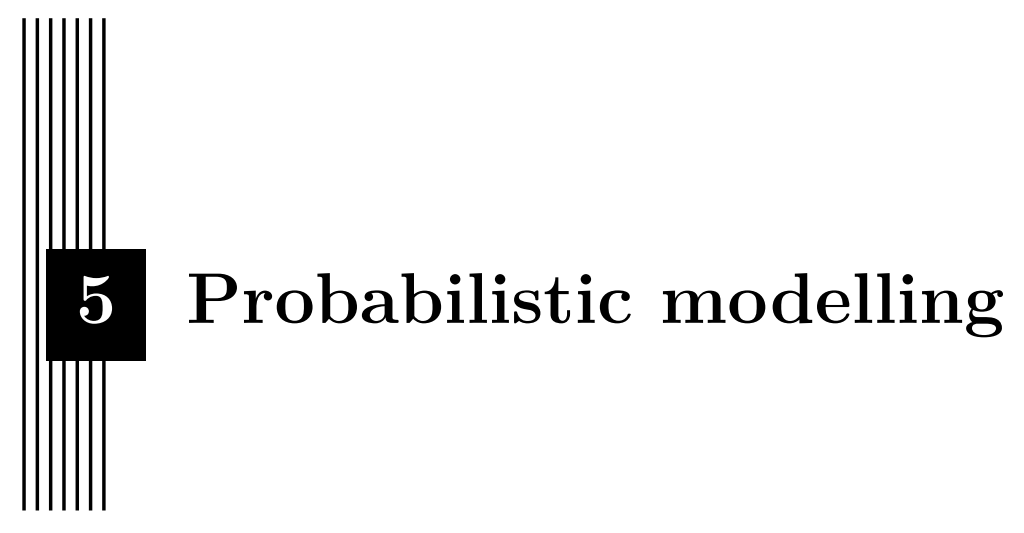

"All models are wrong, but some are useful."

George E. P. Box (1919-2013)

In the previous chapter, we described how the MRI signal is made sensitive to microscopic motion. Making sense of this signal, e.g. to extract information on tissue microstructure, requires modelling. If noise or other sources of uncertainty are involved - which is practically always the case - then probabililty is the natural modelling language. In this chapter we describe probabilistic models for regression that enable uncertainty quantification. Specifically, we will describe Bayesian linear regression and Gaussian process regression, and show that they are actually closely related.

\subsection{Regression models in diffusion MRI}

In regression, we are given pairs of observations of inputs $x$ and corresponding real-valued responses $y$. Based on these we wish to learn a model of the dependency of the response on the inputs, such that the model can make accurate predictions of $y$ for "any" value of $x$.

Regression models, typically parameterized ones, are ubiquitous in applications of dMRI. For instance, regression models are used to describe the directional diffusivity in every voxel, which can be used to trace virtual fibers- 
a procedure referred to as (computational) tractography [22, 42, 55]. Since voxelwise errors accumulate along a fiber, it is reasonable to expect that tractography would benefit greatly from uncertainty quantification [10].

Regression models are also used to derive voxel-based scalar indices such as fractional anisotropy (FA) or return to origin probability (RTOP), as well as the pairwise probability of a pathway being traced between two points. Using such measures it is possible to perform statistical group analyses [104]. For the reliability of such tests it is essential to quantify the uncertainty of the relevant measures - a fact not yet well-established in the field of diffusion MRI.

\subsection{Bayesian linear regression}

In diffusion tensor imaging (DTI) [8], the effective diffusion in a voxel of the human brain is assumed to be (approximately) Gaussian. It is well-established [25, 43] that this assumption is sometimes violated. In particular, voxels in which white matter tracts cross, exhibit more complex diffusion patterns. Quite a few researchers have proposed models that address this limitation of DTI [6, 21, 69, 70, 115, 120]. Several of the most common ones rely on an expansion of the signal in an appropriately chosen functional basis, where the coefficients are determined using least-squares (see table 1 of paper VI). In paper VI we highlight that linear models fitted with least-squares are amenable to a probabilistic reinterpretation. From this interpretion it follows that, under the same assumptions as in the fitting, the least-squares estimate is just the mean of a probability disitribution. More intererestingly, however, we show that it is possible to determine the whole distribution.

\section{The posterior distribution}

In a fixed basis expansion, it is assumed that, in the absence of noise, it would hold that

$$
y(\mathbf{x})=\sum_{i=1}^{d} c_{i} \phi_{i}(\mathbf{x}),
$$

where $y$ is the response variable that we want to model, $\mathbf{x}$ defines a measurement and $\phi_{i}(\mathbf{x})$ are (nonlinear) functions with corresponding coefficients $c_{i}$. In a typical scenario, we are given $n$ observations $\left(\mathbf{x}_{j}, y_{j}\right)$ and the objective is to determine an estimate $\hat{\mathbf{c}}$ such that $\mathbf{y} \approx \Phi \hat{\mathbf{c}}$, where we have introduced the matrix $\Phi_{j i}=\phi_{i}\left(\mathbf{x}_{j}\right)$. Here, we restrict ourselves to one-dimensional measurements, which arguably captures most applications of interest in diffusion MRI, e.g. when the measurement is the echo amplitude.

If we assume that the measurements are contaminated by additive homoscedastic white Gaussian noise, it follows that the likelihood-the proba- 
bility density of the observations given the parameters - is

$$
p(\mathbf{y} \mid \mathbf{c}, \mathbf{x})=\mathcal{N}\left(\Phi \mathbf{c}, \sigma^{2} I\right) .
$$

It is in general possible - and often reasonable - to choose the coefficients such that the likelihood is maximized. When the noise is Gaussian, this yields the least-squares estimate

$$
\begin{aligned}
\hat{\mathbf{c}}_{\mathrm{ML}} & =\underset{\mathbf{c}}{\operatorname{argmin}} \log p(\mathbf{y} \mid \mathbf{c}, \mathbf{x}) \\
& =\underset{\mathbf{c}}{\operatorname{argmin}}\|\mathbf{y}-\Phi \mathbf{c}\|_{2}^{2} \\
& =\left(\Phi^{T} \Phi\right)^{-1} \Phi^{T} \mathbf{y} .
\end{aligned}
$$

One would, however, rather like to know the posterior distribution: the probability density of the coefficients given the data. In the Bayesian formalism, this is obtained using Bayes' rule

$$
p(\mathbf{c} \mid \mathbf{y}, \mathbf{x})=\frac{p(\mathbf{y} \mid \mathbf{c}, \mathbf{x}) p(\mathbf{c})}{p(\mathbf{y} \mid \mathbf{x})} .
$$

The first factor in the nominator is the likelihood, which we have from above. The second factor, the prior, we have to specify ourselves. The denominator can often be determined from the constraint that the posterior distribution needs to integrate to unity.

When specifying a prior, so-called conjugate priors are particularly convenient. This is because, for conjugate priors, the posterior distribution is in the same family as the prior distribution. For the Gaussian likelihood in equation (5.2), a conjugate prior is

$$
p(\mathbf{c})=\mathcal{N}\left(\mathbf{c}_{0}, \Lambda^{-1}\right),
$$

with some known mean $\mathbf{c}_{0}$ and precision (inverse covariance) matrix $\Lambda$. With this choice of likelihood and prior, Bayes' rule can be used to show that the posterior distribution is 13 ]

$$
p(\mathbf{c} \mid \mathbf{y}, \mathbf{x})=\mathcal{N}\left(S^{-1}\left(\sigma^{-2} \Phi^{T} \mathbf{y}+\Lambda \mathbf{c}_{0}\right), S^{-1}\right),
$$

where $S=\sigma^{-2} \Phi^{T} \Phi+\Lambda$. For the Gaussian distribution, the maximum-aposteriori solution - - the mode of the posterior - is the same as its mean. A closer look at the mean reveals that it is the solution of a penalized leastsquares problem,

$$
\begin{aligned}
\mathbf{c}_{\mathrm{MAP}} & =\left(\sigma^{-2} \Phi^{T} \Phi+\Lambda\right)^{-1}\left(\sigma^{-2} \Phi^{T} \mathbf{y}+\Lambda \mathbf{c}_{0}\right)= \\
& =\underset{\mathbf{c}}{\operatorname{argmin}}\{\log p(\mathbf{y} \mid \mathbf{c}, \mathbf{x})+\log p(\mathbf{c})\} \\
& =\underset{\mathbf{c}}{\operatorname{argmin}}\|y-\Phi \mathbf{c}\|_{2}^{2}+\left\|\mathbf{c}-\mathbf{c}_{0}\right\|_{\sigma^{2} \Lambda}^{2} .
\end{aligned}
$$

This is also known as ridge regression or Tikhonov regularization. 


\section{Predictive distribution}

To proceed to making a prediction, the arguably most common approach is to fix the coefficients using e.g. equation (5.9). In contrast, the Bayesian way is to average over all possible coefficients according to the posterior distribution. For brevity, we use $\mathcal{D}$ to denote the observed data $\left(\mathbf{x}_{j}, y_{j}\right)$. Then the predictive distribution for the noise-free response $f_{*}=f\left(\mathbf{x}_{*}\right)$ is given by

$$
\begin{aligned}
p\left(f_{*} \mid \mathcal{D}, \mathbf{x}_{*}\right) & =\int p\left(f_{*} \mid \mathcal{D}, \mathbf{x}_{*}, \mathbf{c}\right) p\left(\mathbf{c} \mid \mathcal{D}, \mathbf{x}_{*}\right) d \mathbf{c} \\
& =\int p\left(f_{*} \mid \mathbf{x}_{*}, \mathbf{c}\right) p(\mathbf{c} \mid \mathbf{y}, \mathbf{x}) d \mathbf{c} .
\end{aligned}
$$

By assumption $f_{*}=\phi\left(\mathbf{x}_{*}\right)^{T} \mathbf{c}$ (for short we will henceforth write $\phi_{*}=\phi\left(\mathbf{x}_{*}\right)$ ), so we may write the first part of the integrand as

$$
p\left(f_{*} \mid \mathbf{x}_{*}, \mathbf{c}\right)=\delta\left(f_{*}-\phi_{*}^{T} \mathbf{c}\right) .
$$

Then, we find that the predictive distribution is

$$
\begin{aligned}
p\left(f_{*} \mid \mathcal{D}, \mathbf{x}_{*}\right) & =\mathcal{N}\left(\mu_{*}, \sigma_{*}^{2}\right), \\
\mu_{*} & =\phi_{*}^{T} S^{-1}\left(\sigma^{-2} \Phi^{T} \mathbf{y}+\Lambda \mathbf{c}_{0}\right), \\
\sigma_{*}^{2} & =\phi_{*}^{T} S^{-1} \phi_{*} .
\end{aligned}
$$

This shows, perhaps reassuringly, that the mean of the predictive distribution is exactly what you get when inserting the maximum-a-posteriori estimate (5.9) for the coefficients in your function fit. However, what this line of reasoning also gives you - almost for free - is a quantification of the uncertainty in the prediction. This is the main result of paper VI, which shows that basically all linear models for diffusion MRI can take advantage of this property.

Incidentally, note that the covariance of the predictions (the uncertainty) (5.15) does not depend on the measurements $\mathbf{y}$. This hints at the possibility of designing, a priori, a measurement scheme that minimizes the predictive uncertainty [32].

\subsection{Gaussian processes}

In paper $\mathrm{V}$, we use Gaussian processes to predict unobserved measurements in a way that makes it possible to reconstruct the ensemble average propagator (cf. section 4.3) with far fewer measurements than ordinarily required. We also propose a reconstruction method that makes use of the uncertainty estimates provided by Gaussian processes. To put these results in context, this section provides an introduction to Gaussian processes.

A Gaussian process is a certain type of stochastic process (cf. section 4.1) that can be thought of as a Gaussian distribution over functions [77]. 
Just as a multivariate Gaussian distribution is fully specified by its mean and covariance matrix, a Gaussian process is fully described by its mean and covariance function. To make this concrete, consider a stochastic variable $f$ distributed according to a multivariate normal distribution with mean $\mu$ and covariance matrix $\Sigma$

$$
f \sim \mathcal{N}(\mu, \Sigma)
$$

The covariance between two points $x_{i}$ and $x_{j}$ are specified by the entry $\Sigma_{i j}$ in the covariance matrix, or if you imagine $\Sigma$ being a function, you could write this as $\Sigma_{i j}=\Sigma\left(x_{i}, x_{j}\right)$.

To make the transition to a Gaussian process, we note that a function $f$ can be loosely thought of as a very long vector, where each entry in the vector $\mathbf{f}(x)$ corresponds to the function value at the input point $x$. Let $\mathbf{x}=\left\{x_{i}\right\}_{i=1}^{N}$ be a vector of uniformly spaced input points, and suppose that $\mathbf{f}(\mathbf{x}) \sim \mathcal{N}(\mu, \Sigma)$ where, as usual, the mean and covariance are defined by

$$
\begin{aligned}
& \boldsymbol{\mu}=\langle\mathbf{f}\rangle \in \mathbb{R}^{N}, \\
& \Sigma=\left\langle(\mathbf{f}-\boldsymbol{\mu})(\mathbf{f}-\boldsymbol{\mu})^{T}\right\rangle \in \mathbb{R}^{N \times N} .
\end{aligned}
$$

In the limit $N \rightarrow \infty$, we get a Gaussian process

$$
f(x) \sim \mathcal{G P}\left(\mu(x), k\left(x, x^{\prime}\right)\right),
$$

where $\mu(x)$ is the mean function and $k\left(x, x^{\prime}\right)$ the covariance function (analogous to an entry in the covariance matrix $\left.\Sigma\left(x, x^{\prime}\right)\right)$. In contrast to the finite dimensional case, the mean and covariance functions are defined for every value of $x$ and $x^{\prime}$. The covariance function tells us how similar two inputs are and thus how much they are allowed to influence each other. The choice of covariance function therefore controls the type of solution we expect, e.g. if it is smooth, periodic or has some particular symmetry. The evaluation $k\left(x_{i}, x_{j}\right)$ of the covariance function at two points, $x_{i}$ and $x_{j}$, is akin to the entry $\Sigma_{i j}$ of a covariance matrix $\Sigma$. We can thus appreciate that for $k\left(x, x^{\prime}\right)$ to be a valid covariance function, any matrix $K$ with elements $k\left(x_{i}, x_{j}\right)$ must be symmetric and positive definite, just like a covariance matrix. Incidentally, this allows us to glean one of the beauties of Gaussian processes: although it involves infinite dimensional objects, it reverts back to "regular" multivariate Gaussian distributions as long as you consider the function values only at a finite set of points.

\section{Gaussian process regression}

Now, let us describe how to use Gaussian processes for regression provided that the mean and covariance functions are known. Suppose that, just as in section 5.2, we have a set of $n$ observations $\mathcal{D}=\left\{\left(\mathbf{x}_{i}, y_{i}\right) \mid i=1, \ldots, n\right\}$ composed of inputs $\mathbf{x}_{i} \in \mathbb{R}^{M}$ and (noisy) responses $y_{i} \in \mathbb{R}^{1}$ generated from a 
latent (unobserved) function $f(\mathbf{x})$ contaminated with additive white Gaussian noise such that $y_{i}=f\left(\mathbf{x}_{i}\right)+\epsilon_{i}$.

Just like in the example with Bayesian linear regression above, Gaussian processes allow you to predict the function value $f_{*}=f\left(\mathbf{x}_{*}\right)$ at an arbitrary input point $\mathbf{x}_{*}$, as well as the corresponding variance $\sigma_{*}^{2}$ (uncertainty if you will). To see how, we organize the inputs as a matrix $X$ with rows $\mathbf{x}_{i}$ and the responses as a vector $\mathbf{y}$. In the following sections, we will discuss the mean- and covariance functions in more detail, however, assuming that they are known, the joint distribution of the training data and the unobserved pair $\left(\mathbf{x}_{*}, f_{*}\right)$ is given by

$$
\left(\begin{array}{c}
\mathbf{y} \\
f_{*}
\end{array}\right) \sim \mathcal{N}_{n+1}\left(\left(\begin{array}{c}
\boldsymbol{\mu}(X) \\
\mu\left(x_{*}\right)
\end{array}\right),\left(\begin{array}{cc}
K+\sigma_{n}^{2} I & \mathbf{k}_{*} \\
\mathbf{k}_{*}^{T} & k_{*}
\end{array}\right)\right),
$$

where $K$ is the $n \times n$ matrix of covariances between all the observed inputs, $\sigma_{n}^{2}$ is the noise variance, $\mathbf{k}_{*}$ is an $n \times 1$ vector of cross-covariances between the observed inputs and $x_{*}$. Finally, $k_{*}=k\left(x_{*}, x_{*}\right)$ is the variance at the point $x_{*}$. We may then use the standard identity for conditioning a Gaussian distribution [13, 77] to find that the conditional probability of $f_{*}$ given $X, \mathbf{y}$ and $x_{*}$, is

$$
\begin{aligned}
p\left(f_{*} \mid X, \mathbf{y}, x_{*}\right) & =\mathcal{N}\left(\mu_{*}, \sigma_{*}^{2}\right), \\
\mu_{*} & =\mu\left(x_{*}\right)+\mathbf{k}_{*}^{T}\left(K+\sigma^{2} I\right)^{-1}(\mathbf{y}-\boldsymbol{\mu}(X)), \\
\sigma_{*}^{2} & =k_{*}-\mathbf{k}_{*}^{T}\left(K+\sigma^{2} I\right)^{-1} \mathbf{k}_{*} .
\end{aligned}
$$

\section{Mean function}

Often, little emphasis is placed on the the mean function; a common practice is to subtract the empirical mean from the data and then assume that the mean function is zero. This is perhaps one of the reasons why Gaussian processes are sometimes touted as being "model free". However, the mean function can be used as a powerful way to encode assumptions into a Gaussian process model [124, 125]. Suppose for instance that a physically plausible, parametric model exists. Would it not be better to estimate the parameters of such a model rather than having a black box function approximator? Well, the good news is you can have it both ways: using the physical model as the mean function of a Gaussian process it is imbued with uncertainty estimates. Most likely, this will result in better and more robust predictions, especially when predicting far from the observations or when extrapolating.

\section{Covariance function}

Since a Gaussian process model is fully specified by its mean and covariance function, and the mean function is typically just the empirical mean of the data, most of the modelling power is in the covariance function. Although 
many covariance functions have been suggested [31, 77], finding new and useful ones is still an active area of research [124]. The most common covariance function is probably the radial basis function kernel

$$
k\left(\mathbf{x}, \mathbf{x}^{\prime}\right)=\exp \left(-\frac{\left\|\mathbf{x}-\mathbf{x}^{\prime}\right\|^{2}}{2 \ell^{2}}\right),
$$

where $\ell^{2}$ is a hyperparameter controlling the lengthscale of the correlations. This kernel, like most popular kernels, is stationary in the same sense as the stochastic process it describes. One can create new kernels by combining existing kernels in ways consistent with a (rather flexible) set of rules [13, 31].

\section{Training a Gaussian Process model}

Given data, a Gaussian process model can be "trained" in order to imitate the characteristics of the data. For a Gaussian process model, training amounts to choosing the hyperparameters $\boldsymbol{\eta}$ such that the marginal likelihood is maximized. This is sometimes referred to as empirical Bayes or type II maximum likelihood. Up to a constant, the logarithm of the marginal likelihood is given by $[77$

$$
\log p(\mathbf{y} \mid \mathbf{x}, \boldsymbol{\eta})=-\frac{1}{2} \tilde{\mathbf{y}}^{T}\left(K+\sigma_{n}^{2} I\right)^{-1} \tilde{\mathbf{y}}-\frac{1}{2} \log \left|K+\sigma_{n}^{2} I\right|,
$$

where $\tilde{\mathbf{y}}=\mathbf{y}-\boldsymbol{\mu}(X)$.

\subsection{The connection between Bayesian linear regression and Gaussian processes}

We will now elicit how Bayesian linear regression fit within the framework of Gaussian processes. To this end, we define the vector $\mathbf{k}_{*}=\Phi \Lambda^{-1} \boldsymbol{\phi}_{*}$ and the matrix $K=\Phi \Lambda^{-1} \Phi^{T}$. Recalling the definition $S=\sigma^{-2} \Phi^{T} \Phi+\Lambda$, we may rewrite the predictive mean, equation (5.14), as follows

$$
\begin{aligned}
\mu_{*} & =\phi_{*}^{T} S^{-1}\left(\sigma^{-2} \Phi^{T} \mathbf{y}+\Lambda \mathbf{c}_{0}\right) \\
& =\phi_{*}^{T} S^{-1} \underbrace{\sigma^{-2} \Phi^{T}\left(K+\sigma^{2} I\right)}_{S \Lambda^{-1} \Phi^{T}}\left(K+\sigma^{2} I\right)^{-1} \mathbf{y}+\phi_{*}^{T} S^{-1} \Lambda \mathbf{c}_{0} \\
& =\underbrace{\phi_{*}^{T} \Lambda^{-1} \Phi^{T}}_{\mathbf{k}_{*}^{T}}\left(K+\sigma^{2} I\right)^{-1} \mathbf{y}+\phi_{*}^{T} S^{-1} \Lambda \mathbf{c}_{0} \\
& =\mathbf{k}_{*}^{T}\left(K+\sigma^{2} I\right)^{-1} \mathbf{y}+\boldsymbol{\phi}_{*}^{T}\left(\Lambda^{-1}-\Lambda^{-1} \Phi^{T}(\sigma^{2} I+\underbrace{\Phi \Lambda^{-1} \Phi^{T}}_{K})^{-1} \Phi \Lambda^{-1}\right) \Lambda \mathbf{c}_{0} \\
& =\boldsymbol{\phi}_{*}^{T} \mathbf{c}_{0}+\mathbf{k}_{*}^{T}\left(K+\sigma^{2} I\right)^{-1}\left(\mathbf{y}-\Phi \mathbf{c}_{0}\right),
\end{aligned}
$$


where we used the matrix inversion lemma in equation (5.29). In a similar way it is possible to rewrite the predictive variance (5.15) as

$$
\sigma_{*}^{2}=\phi_{*}^{T} \Lambda^{-1} \phi_{*}+\mathbf{k}_{*}^{T}\left(K+\sigma^{2} I\right)^{-1} \mathbf{k}_{*} .
$$

Comparing these formulations of the predictive mean (5.30) and variance (5.31) to the ones for Gaussian process regression (5.22, 5.23), we may identify

$$
\begin{aligned}
\mu(\mathbf{x}) & =\phi(\mathbf{x})^{T} \mathbf{c}_{0}, \\
k\left(\mathbf{x}, \mathbf{x}^{\prime}\right) & =\phi(\mathbf{x})^{T} \Lambda^{-1} \phi\left(\mathbf{x}^{\prime}\right) .
\end{aligned}
$$

In other words, Bayesian linear regression is equivalent to Gaussian process regression with this particular choice of mean- and covariance functions. Computationally, the formulation as a Gaussian process is more efficient than ordinary Bayesian linear regression if there are more basis functions than observations.

Interestingly, in equations $(5.30)$ and $(5.31)$ the inputs $\mathbf{x}$ only enter through the mean- and covariance functions. If there are a closed-form expressions for those, then all calculations can be done without ever evaluating the basis functions. This suggests the Gaussian process approach to regression: rather than introducing a set of basis functions, which implicitly define a mean- and covariance function, we define the mean- and covariance functions directly. 


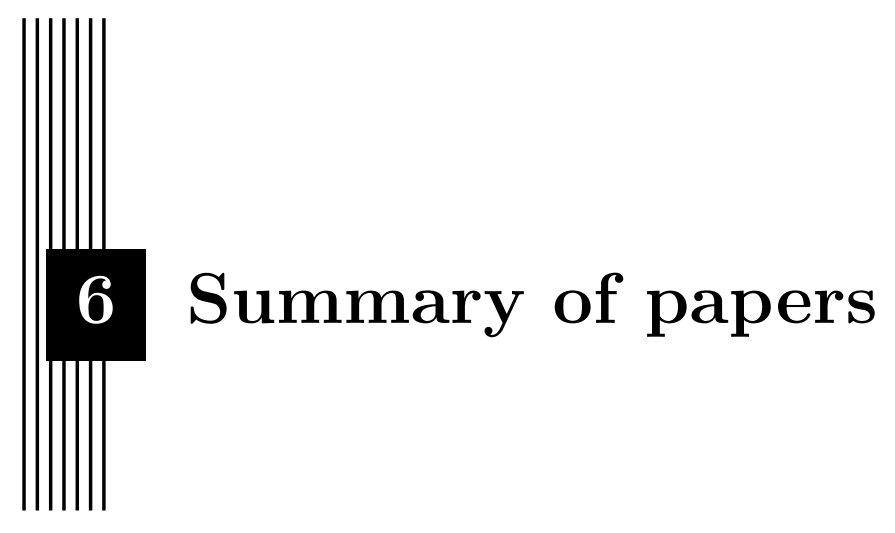

"Do. Or do not. There is no try."

Yoda

In this chapter, we review the research questions put forth in the introduction (chapter 1). For each research question, we summarize the most relevant papers included in the thesis. This is to put the papers in context and to clarify how they align with the overall aim of the thesis. For each paper, my contributions are stated explicitly.

\section{Is it possible, using only MR images, to derive a substitute to CT images for use in radiotherapy?}

Radiotherapy planning requires simulation of radiation transport. The necessary physical properties are typically derived from computed tomography (CT) images, but in some cases only magnetic resonance (MR) images are available. A common but somewhat simplistic solution to this problem is to approximate all tissue properties as equivalent to those of water.

In paper II, we improve upon this approximation by automatically segmenting the skull. The segmentation is done by a machine learning algorithm that has been trained on patients with paired MR and CT images, and uses a combination of local and global information as input. My contributions were 
to prepare the data, create the model, implement it in computer code and write the paper.

In paper III, we further refine the CT substitute, which we refer to as a pseudo-CT. We demonstrate that a realistic, patient-specific, pseudo-CT of the head can be derived from an anatomical MR image using a method we refer to as atlas-based regression. Given a target MR and an atlas database comprising MR and CT pairs, atlas-based regression works by registering (aligning) each atlas MR to the target MR, applying the resulting displacement fields to the corresponding atlas CTs and, finally, fusing the deformed atlas CTs into a single pseudo-CT. My contributions were to formulate the research goal, prepare the data, create the model, implement it in computer code and write the paper.

\section{How can we make diffusion MRI with continuous gradient waveforms viable on clinical MR systems?}

Diffusion MRI is a useful probe of tissue microstructure, which is already used to derive biomarkers that inform radiotherapy treatments. Recent developments, in particular diffusion measurements using continuous gradient waveforms, have shown that there is yet more information to uncover. However, for such diffusion measurements to reach clinical use, it must be possible to perform them on standard clinical MR systems.

In paper III, we formulate a constrained optimization problem that maximizes the signal strength in a diffusion measurement with continuous gradient waveforms, while respecting the constraints imposed by the MR system. The method's efficacy and flexibility is demonstrated both experimentally and by theoretical comparisons with previous work. The code, together with a graphical user interface, is available online 1 . My contributions were to create the model, implement it in computer code and write the paper.

In paper IV], we demonstrate that it is technically feasible - on a range of clinical MRI systems - to perform a certain type of diffusion measurement with continuous gradient waveforms called diffusional variance decomposition (DIVIDE). The imaging protocols were designed using the optimization method from paper III. We assessed the technical feasibility based on signal characteristics and quality of parameter maps in a single volunteer scanned at all systems. My contributions were to refine the optimization software, support its use and to review and edit the paper.

\footnotetext{
${ }^{1}$ https://github.com/jsjol/NOW
} 


\section{Is it possible to develop or reinterpret existing diffusion MRI models in a way that allows uncertainty quantification?}

By fitting a model to diffusion MRI measurements it is possible to derive various quantitative features, and to perform white matter tractography. However, because of measurement noise and the fact that no model is perfect, any estimate is uncertain to some extent. The question is: how much?

In paper $\mathrm{V}$, we describe a flexible model that uses Gaussian processes to represent the signal. We show that by using this model it is possible to estimate something called the ensemble averaged propagator (EAP) with much fewer measurements than what would ordinarily be required. Moreover, Gaussian process models such as ours come with a rigourous way of reasoning about uncertainty. We describe how the uncertainty can be incorporated in the estimation of the EAP. My contributions were to formulate the research goal, create the model, implement it in computer code and write the paper.

In paper $\mathrm{VI}$, we present a probabilistic reinterpretation of some of the most popular diffusion MRI models. Ordinarily, these models lack any notion of uncertainty, but our reinterpretation makes it possible to quantify the uncertainty of any derived quantity. In particular, for quantities that are affine functions of the coefficients, the posterior distribution can be expressed in closed-form. We validated the uncertainty quantification on simulated data and also showed that it was possible to use on in vivo data. My contributions were to formulate the research goal, create the model, implement it in computer code and write the paper. 



\section{Bibliography}

[1] A. Abragam. The Principles of Nuclear Magnetism. International series of monographs on physics. Clarendon Press, 1961.

[2] Anders Ahnesjö. "Collapsed cone convolution of radiant energy for photon dose calculation in heterogeneous media". In: Medical physics 16.4 (1989), pp. 577-592.

[3] Anders Ahnesjö and Maria Mania Aspradakis. "Dose calculations for external photon beams in radiotherapy". In: Physics in Medicine and Biology 44.11 (1999), R99-R155.

[4] Anders Ahnesjö, Mikael Saxner, and Avo Trepp. "A pencil beam model for photon dose calculation". In: Medical physics 19.2 (1992), pp. 263273.

[5] Bruce Alberts, Dennis Bray, Karen Hopkin, Alexander Johnson, Julian Lewis, Martin Raff, Keith Roberts, and Peter Walter. Essential cell biology. 3rd. Garland Science, 2013.

[6] Daniel C Alexander. "Multiple-fiber reconstruction algorithms for diffusion MRI". In: Annals of the New York Academy of Sciences 1064.1 (2005), pp. 113-133.

[7] David W Andrews, Charles B Scott, Paul W Sperduto, Adam E Flanders, Laurie E Gaspar, Michael C Schell, Maria Werner-Wasik, William Demas, Janice Ryu, Jean-Paul Bahary, et al. "Whole brain radiation therapy with or without stereotactic radiosurgery boost for patients with one to three brain metastases: phase III results of the RTOG 9508 randomised trial". In: The Lancet 363.9422 (2004), pp. 1665-1672. 
[8] Peter J Basser, James Mattiello, and Denis LeBihan. "MR diffusion tensor spectroscopy and imaging." In: Biophysical journal 66.1 (1994), p. 259 .

[9] Christian Beaulieu. "The basis of anisotropic water diffusion in the nervous system-a technical review". In: NMR in Biomedicine 15.7-8 (2002), pp. 435-455.

[10] Timothy EJ Behrens, H Johansen Berg, Saad Jbabdi, Matthew FS Rushworth, and Mark W Woolrich. "Probabilistic diffusion tractography with multiple fibre orientations: What can we gain?" In: Neuroimage 34.1 (2007), pp. 144-155.

[11] Stanley H Benedict, Kamil M Yenice, David Followill, James M Galvin, William Hinson, Brian Kavanagh, Paul Keall, Michael Lovelock, Sanford Meeks, Lech Papiez, et al. "Stereotactic body radiation therapy: the report of AAPM Task Group 101". In: Medical physics 37.8 (2010), pp. 4078-4101.

[12] Jacques Bernier, Eric J Hall, and Amato Giaccia. "Radiation oncology: a century of achievements". In: Nature Reviews Cancer 4.9 (2004), pp. $737-747$.

[13] Christopher M Bishop. Pattern recognition and machine learning. springer, 2006.

[14] J Martin Brown, David J Carlson, and David J Brenner. "The tumor radiobiology of SRS and SBRT: are more than the 5 R's involved?" In: International Journal of Radiation Oncology* Biology* Physics 88.2 (2014), pp. 254-262.

[15] Paul T Callaghan. Translational dynamics and magnetic resonance: principles of pulsed gradient spin echo NMR. Oxford University Press, 2011.

[16] Subrahmanyan Chandrasekhar. "Stochastic problems in physics and astronomy". In: Reviews of modern physics 15.1 (1943), p. 1.

[17] L Chen, RA Price Jr, TB Nguyen, L Wang, JS Li, L Qin, M Ding, E Palacio, CM Ma, and A Pollack. "Dosimetric evaluation of MRI-based treatment planning for prostate cancer". In: Physics in medicine and biology 49.22 (2004), p. 5157.

[18] William T Coffey and Yuri P Kalmykov. The Langevin equation: with applications to stochastic problems in physics, chemistry and electrical engineering. World Scientific, 2004.

[19] J Deacon, MJ Peckham, and GG Steel. "The radioresponsiveness of human tumours and the initial slope ofthe cell survival curve". In: $R a$ diotherapy and Oncology 2.4 (1984), pp. 317-323.

[20] Manuel Delgado. "Classroom Note: The Lagrange-Charpit Method". In: SIAM review 39.2 (1997), pp. 298-304. 
[21] Maxime Descoteaux, Elaine Angelino, Shaun Fitzgibbons, and Rachid Deriche. "Regularized, fast, and robust analytical Q-ball imaging". In: Magnetic resonance in medicine 58.3 (2007), pp. 497-510.

[22] Maxime Descoteaux, Rachid Deriche, Thomas R Knosche, and Alfred Anwander. "Deterministic and probabilistic tractography based on complex fibre orientation distributions". In: IEEE transactions on medical imaging 28.2 (2009), pp. 269-286.

[23] Albert Einstein. "Über die von der molekularkinetischen Theorie der Wärme geforderte Bewegung von in ruhenden Flüssigkeiten suspendierten Teilchen". In: Annalen der physik 322.8 (1905), pp. 549560 .

[24] Anders Eklund. "Signal Processing for Robust and Real-Time fMRI With Application to Brain Computer Interfaces". Licentiate thesis. Linköping University Electronic Press, 2010.

[25] Shawna Farquharson, J-Donald Tournier, Fernando Calamante, Gavin Fabinyi, Michal Schneider-Kolsky, Graeme D Jackson, and Alan Connelly. "White matter fiber tractography: why we need to move beyond DTI". In: Journal of neurosurgery 118.6 (2013), pp. 1367-1377.

[26] Gerald Feinberg. "Possibility of faster-than-light particles". In: Physical Review 159.5 (1967), p. 1089.

[27] Bernard Fertil and Edmond-Philippe Malaise. "Inherent cellular radiosensitivity as a basic concept for human tumor radiotherapy". In: International Journal of Radiation Oncology* Biology* Physics 7.5 (1981), pp. 621-629.

[28] Matthias Fippel. "Fast Monte Carlo dose calculation for photon beams based on the VMC electron algorithm". In: Medical Physics 26.8 (1999), pp. 1466-1475.

[29] Domenico Formica and Sergio Silvestri. "Biological effects of exposure to magnetic resonance imaging: an overview". In: Biomedical engineering online 3.1 (2004), p. 11.

[30] Monica Garcia-Barros, Francois Paris, Carlos Cordon-Cardo, David Lyden, Shahin Rafii, Adriana Haimovitz-Friedman, Zvi Fuks, and Richard Kolesnick. "Tumor response to radiotherapy regulated by endothelial cell apoptosis". In: Science 300.5622 (2003), pp. 1155-1159.

[31] Marc G Genton. "Classes of kernels for machine learning: a statistics perspective". In: Journal of machine learning research 2.Dec (2001), pp. 299-312.

[32] Alex Gorodetsky and Youssef Marzouk. "Mercer kernels and integrated variance experimental design: Connections between Gaussian process regression and polynomial approximation". In: SIAM/ASA Journal on Uncertainty Quantification 4.1 (2016), pp. 796-828. 
[33] Louis Harold Gray, A__D Conger, M Ebert, S Hornsey, and OCA Scott. "The concentration of oxygen dissolved in tissues at the time of irradiation as a factor in radiotherapy". In: The British journal of radiology 26.312 (1953), pp. 638-648.

[34] Denis S Grebenkov. "Use, misuse, and abuse of apparent diffusion coefficients". In: Concepts in Magnetic Resonance Part A 36.1 (2010), pp. 24-35.

[35] David J. Griffiths. Introduction to quantum mechanics. 2nd. Pearson Education International, 2005.

[36] E. Mark Haacke, Robert W. Brown, Michael R. Thompson, and Ramesh Venkatesan. Magnetic resonance imaging: physical principles and sequence design. Wiley-Liss, 1999.

[37] Erwin L Hahn. "Spin echoes". In: Physical Review 80.4 (1950), p. 580.

[38] Eric J Hall and Amato J Giaccia. Radiobiology for the Radiologist. Lippincott Williams \& Wilkins, 2006.

[39] Magnus Herberthson, Evren Özarslan, Hans Knutsson, and CarlFredrik Westin. "Dynamics of local magnetization in the eigenbasis of the Bloch-Torrey operator". In: The Journal of Chemical Physics 146.12 (2017), p. 124201.

[40] Sami Hissoiny, Benôt Ozell, Hugo Bouchard, and Philippe Després. "GPUMCD: A new GPU-oriented Monte Carlo dose calculation platform". In: Medical physics 38.2 (2011), pp. 754-764.

[41] Michael Höckel and Peter Vaupel. "Tumor hypoxia: definitions and current clinical, biologic, and molecular aspects". In: Journal of the National Cancer Institute 93.4 (2001), pp. 266-276.

[42] Brian J Jellison, Aaron S Field, Joshua Medow, Mariana Lazar, M Shariar Salamat, and Andrew L Alexander. "Diffusion tensor imaging of cerebral white matter: a pictorial review of physics, fiber tract anatomy, and tumor imaging patterns". In: American Journal of Neuroradiology 25.3 (2004), pp. 356-369.

[43] Ben Jeurissen, Alexander Leemans, Jacques-Donald Tournier, Derek K Jones, and Jan Sijbers. "Investigating the prevalence of complex fiber configurations in white matter tissue with diffusion magnetic resonance imaging". In: Human brain mapping 34.11 (2013), pp. 2747-2766.

[44] Bing Jian, Baba C Vemuri, Evren Özarslan, Paul R Carney, and Thomas H Mareci. "A novel tensor distribution model for the diffusionweighted MR signal”. In: NeuroImage 37.1 (2007), pp. 164-176.

[45] Hongyu Jiang, Joao Seco, and Harald Paganetti. "Effects of Hounsfield number conversion on CT based proton Monte Carlo dose calculations". In: Medical physics 34.4 (2007), pp. 1439-1449. 
[46] Mark Kac. "On distributions of certain Wiener functionals". In: Transactions of the American Mathematical Society 65.1 (1949), pp. 1-13.

[47] Mark Kac et al. "On some connections between probability theory and differential and integral equations". In: Proceedings of the second Berkeley symposium on mathematical statistics and probability. The Regents of the University of California. 1951.

[48] Olav Kallenberg. Foundations of modern probability. 2nd ed. SpringerVerlag New York, 2002.

[49] Iwan Kawrakow, Matthias Fippel, and Klaus Friedrich. "3D electron dose calculation using a Voxel based Monte Carlo algorithm (VMC)". In: Medical Physics 23.4 (1996), pp. 445-457.

[50] Douglas Kondziolka, Samuel M Shin, Andrew Brunswick, Irene Kim, and Joshua S Silverman. "The biology of radiosurgery and its clinical applications for brain tumors". In: Neuro-oncology 17.1 (2015), pp. 2944 .

[51] Thomas Krieger and Otto A Sauer. "Monte Carlo-versus pencil-beam/collapsed-cone-dose calculation in a heterogeneous multi-layer phantom". In: Physics in medicine and biology 50.5 (2005), p. 859.

[52] Rep Kubo. "The fluctuation-dissipation theorem". In: Reports on progress in physics 29.1 (1966), p. 255.

[53] Jan JW Lagendijk, Bas W Raaymakers, Alexander JE Raaijmakers, Johan Overweg, Kevin J Brown, Ellen M Kerkhof, Richard W van der Put, Björn Hårdemark, Marco van Vulpen, and Uulke A van der Heide. "MRI/linac integration". In: Radiotherapy and Oncology 86.1 (2008), pp. 25-29.

[54] Paul Langevin. "Sur la théorie du mouvement brownien". In: CR Acad. Sci. Paris 146.530-533 (1908), p. 530.

[55] Denis Le Bihan. "Looking into the functional architecture of the brain with diffusion MRI". In: Nature Reviews Neuroscience 4.6 (2003), pp. $469-480$.

[56] Lars Leksell. "The stereotaxic method and radiosurgery of the brain". In: Acta Chir. Scand. 102 (1951), pp. 316-319.

[57] Zhi-Pei Liang and Paul C Lauterbur. Principles of magnetic resonance imaging. SPIE Optical Engineering Press, 2000.

[58] Mark E Linskey, David W Andrews, Anthony L Asher, Stuart H Burri, Douglas Kondziolka, Paula D Robinson, Mario Ammirati, Charles S Cobbs, Laurie E Gaspar, Jay S Loeffler, et al. "The role of stereotactic radiosurgery in the management of patients with newly diagnosed brain metastases: a systematic review and evidence-based clinical practice guideline". In: Journal of neuro-oncology 96.1 (2010), pp. 45-68. 
[59] L Dade Lunsford, Douglas Kondziolka, John C Flickinger, David J Bissonette, Charles A Jungreis, Ann H Maitz, Joseph A Horton, and Robert J Coffey. "Stereotactic radiosurgery for arteriovenous malformations of the brain". In: Journal of neurosurgery 75.4 (1991), pp. 512524 .

[60] Satoshi Maesawa, Camille Salame, John C Flickinger, Stephen Pirris, Douglas Kondziolka, and L Dade Lunsford. "Clinical outcomes after stereotactic radiosurgery for idiopathic trigeminal neuralgia". In: Journal of neurosurgery 94.1 (2001), pp. 14-20.

[61] Jan R Magnus and Heinz Neudecker. "The commutation matrix: some properties and applications". In: The Annals of Statistics (1979), pp. 381-394.

[62] Umberto Marini Bettolo Marconi, Andrea Puglisi, Lamberto Rondoni, and Angelo Vulpiani. "Fluctuation-dissipation: response theory in statistical physics". In: Physics reports 461.4-6 (2008), pp. 111-195.

[63] Naruhiro Matsufuji, Hiromi Tomura, Yasuyuki Futami, Haruo Yamashita, Akio Higashi, Shinichi Minohara, Masahiro Endo, and Tatsuaki Kanai. "Relationship between CT number and electron density, scatter angle and nuclear reaction for hadron-therapy treatment planning". In: Physics in medicine and biology 43.11 (1998), p. 3261.

[64] Sören Mattsson, Anders Brahme, Jörgen Carlsson, Juliana Denekamp, Eva Forssell-Aronsson, Mikael Hellström, Karl-Axel Johansson, Elisabeth Kjellén, Bo Littbrand, Bo Nordenskjöld, et al. "Swedish Cancer Society radiation therapy research investigation". In: Acta Oncologica 41.7 (2002), pp. 596-603.

[65] Donald W McRobbie, Elizabeth A Moore, Martin J Graves, and Martin R Prince. MRI from Picture to Proton. Cambridge university press, 2006.

[66] Johanna Skarpman Munter and Jens Sjölund. "Dose-volume histogram prediction using density estimation". In: Physics in medicine and biology 60.17 (2015), pp. 6923-6936. DOI: 10.1088/0031-9155/60/17/ 6923.

[67] Edward Nelson. "Feynman integrals and the Schrödinger equation". In: Journal of Mathematical Physics 5.3 (1964), pp. 332-343.

[68] Anders Norlund. "Costs of radiotherapy". In: Acta Oncologica 42.5-6 (2003), pp. 411-415.

[69] Evren Özarslan, Cheng Guan Koay, Timothy M Shepherd, Michal E Komlosh, M Okan İrfanoğlu, Carlo Pierpaoli, and Peter J Basser. "Mean apparent propagator (MAP) MRI: a novel diffusion imaging method for mapping tissue microstructure". In: NeuroImage 78 (2013), pp. $16-32$. 
[70] Evren Özarslan and Thomas H Mareci. "Generalized diffusion tensor imaging and analytical relationships between diffusion tensor imaging and high angular resolution diffusion imaging". In: Magnetic resonance in Medicine 50.5 (2003), pp. 955-965.

[71] Evren Özarslan, Baba C Vemuri, and Thomas H Mareci. "Generalized scalar measures for diffusion MRI using trace, variance, and entropy". In: Magnetic resonance in Medicine 53.4 (2005), pp. 866-876.

[72] Heon Joo Park, Robert J Griffin, Susanta Hui, Seymour H Levitt, and Chang W Song. "Radiation-induced vascular damage in tumors: implications of vascular damage in ablative hypofractionated radiotherapy (SBRT and SRS)". In: Radiation research 177.3 (2012), pp. 311-327.

[73] Photon, Electron, Proton and Neutron Interaction Data for Body Tissues. Report 46. International Commission on Radiation Units and Measurements. 1992.

[74] R Prabhakar, PK Julka, T Ganesh, A Munshi, RC Joshi, and GK Rath. "Feasibility of using MRI alone for 3D radiation treatment planning in brain tumors". In: Japanese journal of clinical oncology 37.6 (2007), pp. $405-411$.

[75] S James Press. Applied multivariate analysis: using Bayesian and frequentist methods of inference. 2nd ed. Dover, 2005.

[76] Kevin M Prise, Giuseppe Schettino, Melvyn Folkard, and Kathryn D Held. "New insights on cell death from radiation exposure". In: The lancet oncology 6.7 (2005), pp. 520-528.

[77] Carl Edward Rasmussen and Christopher KI Williams. Gaussian processes for machine learning. Vol. 1. MIT press Cambridge, 2006.

[78] Report from SSM's scientific council on ionizing radiation within oncology, 2010. Report 2011:25. Swedish Radiation Safety Authority. 2011.

[79] Report from SSM's scientific council on ionizing radiation within oncology, 2013. MR in radiotherapy - an important step towards personalised treatment? Report 2014:51. Swedish Radiation Safety Authority. 2014.

[80] John P. Ridgway. "Gradient Echo Versus Spin Echo". In: Cardiovascular MR Manual. Springer, Cham, 2015.

[81] Ulrik Ringborg, David Bergqvist, Bengt Brorsson, Eva Cavallin-Ståhl, Jeanette Ceberg, Nina Einhorn, Jan-erik Frödin, Johannes Järhult, Gunilla Lamnevik, Christer Lindholm, et al. "The Swedish Council on Technology Assessment in Health Care (SBU) systematic overview of radiotherapy for cancer including a prospective survey of radiotherapy practice in Sweden 2001-summary and conclusions". In: Acta oncologica 42.5-6 (2003), pp. 357-365. 
[82] Hannes Risken. The Fokker-Planck equation: Methods of Solution and Applications. 2nd ed. Springer Series in Synergetics. Springer-Verlag Berlin Heidelberg, 1996.

[83] Matthew D Robson, Peter D Gatehouse, Mark Bydder, and Graeme M Bydder. "Magnetic resonance: an introduction to ultrashort TE (UTE) imaging". In: Journal of computer assisted tomography 27.6 (2003), pp. 825-846.

[84] Wilfried Schneider, Thomas Bortfeld, and Wolfgang Schlegel. "Correlation between CT numbers and tissue parameters needed for Monte Carlo simulations of clinical dose distributions". In: Physics in medicine and biology 45.2 (2000), p. 459.

[85] K Schulten and I Kosztin. Lectures in theoretical biophysics. University of Illinois, 2000.

[86] Joao Seco and PM Evans. "Assessing the effect of electron density in photon dose calculations". In: Medical physics 33.2 (2006), pp. 540-552.

[87] Joao Seco and Frank Verhaegen. Monte Carlo techniques in radiation therapy. CRC Press, 2013.

[88] Ashoke Sen. "Rolling tachyon". In: Journal of High Energy Physics 2002.04 (2002), p. 048.

[89] Joakim da Silva, Richard Ansorge, and Rajesh Jena. "Fast pencil beam dose calculation for proton therapy using a double-Gaussian beam model". In: Frontiers in oncology 5 (2015).

[90] Joakim da Silva, Richard Ansorge, and Rajesh Jena. "Sub-second pencil beam dose calculation on GPU for adaptive proton therapy". In: Physics in medicine and biology 60.12 (2015), p. 4777.

[91] Jens Sjölund. Method And System For Calibration. Patent Application PCT/EP2014/057659. Priority date 2014-04-15.

[92] Jens Sjölund. Radiotherapy planning systems. Patent Application PCT/EP2016/079560. Priority date 2015-12-04.

[93] Jens Sjölund. Systems and methods for optimized treatment planning. United States Patent Application US20170177812 A1. Priority date 2015-12-25.

[94] Jens Sjölund, Anders Eklund, Evren Özarslan, Magnus Herberthson, Maria Bånkestad, and Hans Knutsson. "Bayesian uncertainty quantification in linear models for diffusion MRI". In: arXiv preprint arXiv:1711.06002 (2017). Submitted for publication. 
[95] Jens Sjölund, Anders Eklund, Evren Özarslan, and Hans Knutsson. "Gaussian process regression can turn non-uniform and undersampled diffusion MRI data into diffusion spectrum imaging". In: 14th International Symposium on Biomedical Imaging (ISBI). IEEE. 2017, pp. 778782. DOI: 10.1109/ISBI .2017.7950634.

[97] Jens Sjölund, Daniel Forsberg, Mats Andersson, and Hans Knutsson. "Generating patient specific pseudo-CT of the head from MR using atlas-based regression". In: Physics in Medicine and Biology 60.2 (2015), pp. 825-839. DOI: 10.1088/0031-9155/60/2/825.

[98] Jens Sjölund and Xiao Han. System And Method For Automatic Treatment Planning. United States Patent Application US20150367145 A1. Priority date 2014-06-18.

[99] Jens Sjölund, Andreas Eriksson Järliden, Mats Andersson, Hans Knutsson, and Håkan Nordström. "Skull Segmentation in MRI by a Support Vector Machine Combining Local and Global Features". In: 22nd International Conference on Pattern Recognition (ICPR). IEEE. 2014, pp. 3274-3279. DOI: 10.1109/ICPR.2014.564.

[101] Jens Sjölund, Markus Nilsson, Daniel Topgaard, Carl-Fredrik Westin, and Hans Knutsson. "Constrained optimization of gradient waveforms for isotropic diffusion encoding". In: Proceedings of the International Society of Magnetic Resonance in Medicine. 2015.

[102] Jens Sjölund and Håkan Nordström. Convex inverse planning method. United States Patent Application US 2017xxxxxxx A1. Priority date 2017-04-28.

[103] Jens Sjölund, Filip Szczepankiewicz, Markus Nilsson, Daniel Topgaard, Carl-Fredrik Westin, and Hans Knutsson. "Constrained optimization of gradient waveforms for generalized diffusion encoding". In: Journal of Magnetic Resonance 261 (2015), pp. 157-168. DOI: 10.1016/j.jmr. 2015.10.012.

[104] Stephen M Smith, Mark Jenkinson, Heidi Johansen-Berg, Daniel Rueckert, Thomas E Nichols, Clare E Mackay, Kate E Watkins, Olga Ciccarelli, M Zaheer Cader, Paul M Matthews, et al. "Tract-based spatial statistics: voxelwise analysis of multi-subject diffusion data". In: Neuroimage 31.4 (2006), pp. 1487-1505.

[105] G Gordon Steel, Trevor J McMillan, and JH Peacock. "The 5Rs of radiobiology". In: International journal of radiation biology 56.6 (1989), pp. 1045-1048.

[106] EO Stejskal and JE Tanner. "Spin diffusion measurements: spin echoes in the presence of a time-dependent field gradient". In: The journal of chemical physics 42.1 (1965), pp. 288-292. 
[107] SD Stoller, William Happer, and Freeman J Dyson. "Transverse spin relaxation in inhomogeneous magnetic fields". In: Physical Review A 44.11 (1991), p. 7459.

[108] Hans Svensson and Torgil R Möller. "Developments in radiotherapy". In: Acta Oncologica 42.5-6 (2003), pp. 430-442.

[109] Filip Szczepankiewicz, Jens Sjölund, Freddy Ståhlberg, Jimmy Lätt, and Markus Nilsson. "Whole-brain diffusional variance decomposition (DIVIDE) in 8 minutes: Technical feasibility at 1.5, 3, and 7 T". In: Proceedings of the International Society of Magnetic Resonance in Medicine. 2017.

[110] Filip Szczepankiewicz, Jens Sjölund, Freddy Ståhlberg, Jimmy Lätt, and Markus Nilsson. "Whole-brain diffusional variance decomposition (DIVIDE): Demonstration of technical feasibility at clinical MRI systems". In: arXiv preprint arXiv:1612.06741 (2016). In preparation.

[111] Mats Talbäck and Paul W Dickman. "Predicting the survival of cancer patients recently diagnosed in Sweden and an evaluation of predictions published in 2004". In: Acta Oncologica 51.1 (2012), pp. 17-27.

[112] Mats Talbäck, Måns Rosén, Magnus Stenbeck, and Paul W Dickman. "Cancer patient survival in Sweden at the beginning of the third millennium-predictions using period analysis". In: Cancer Causes 8 Control 15.9 (2004), pp. 967-976.

[113] Tissue Substitutes in Radiation Dosimetry and Measurement. Report 44. International Commission on Radiation Units and Measurements. 1989 .

[114] Henry C Torrey. "Bloch equations with diffusion terms". In: Physical Review 104.3 (1956), p. 563.

[115] David S Tuch, Timothy G Reese, Mette R Wiegell, Nikos Makris, John W Belliveau, and Van J Wedeen. "High angular resolution diffusion imaging reveals intravoxel white matter fiber heterogeneity". In: Magnetic resonance in medicine 48.4 (2002), pp. 577-582.

[116] Nicolaas Godfried Van Kampen. Stochastic processes in physics and chemistry. 3rd. North Holland, 2007.

[117] Peter Vaupel. "Tumor microenvironmental physiology and its implications for radiation oncology". In: Seminars in radiation oncology. Vol. 14. 3. Elsevier. 2004, pp. 198-206.

[118] MA Vermoolen, TC Kwee, and RAJ Nievelstein. "Apparent diffusion coefficient measurements in the differentiation between benign and malignant lesions: a systematic review". In: Insights into imaging 3.4 (2012), pp. 395-409.

[119] John F Ward. "Nature of lesions formed by ionizing radiation". In: DNA damage and repair. Springer, 1998, pp. 65-84. 
[120] Van J Wedeen, Patric Hagmann, Wen-Yih Isaac Tseng, Timothy G Reese, and Robert M Weisskoff. "Mapping complex tissue architecture with diffusion spectrum magnetic resonance imaging". In: Magnetic resonance in medicine 54.6 (2005), pp. 1377-1386.

[121] Markus Weiger, David O Brunner, Benjamin E Dietrich, Colin F Müller, and Klaas P Pruessmann. "ZTE imaging in humans". In: Magnetic Resonance in Medicine 70.2 (2013), pp. 328-332.

[122] Carl-Fredrik Westin, Hans Knutsson, Ofer Pasternak, Filip Szczepankiewicz, Evren Özarslan, Danielle van Westen, Cecilia Mattisson, Mats Bogren, Lauren J O'donnell, Marek Kubicki, et al. "Q-space trajectory imaging for multidimensional diffusion MRI of the human brain". In: Neuroimage 135 (2016), pp. 345-362.

[123] Florian Wiesinger, Laura I Sacolick, Anne Menini, Sandeep S Kaushik, Sangtae Ahn, Patrick Veit-Haibach, Gaspar Delso, and Dattesh D Shanbhag. "Zero TE MR bone imaging in the head". In: Magnetic resonance in medicine 75.1 (2016), pp. 107-114.

[124] Andrew Gordon Wilson. "Covariance kernels for fast automatic pattern discovery and extrapolation with Gaussian processes". PhD thesis. University of Cambridge, 2014.

[125] Josef Wilzen, Anders Eklund, and Mattias Villani. "Physiological Gaussian Process Priors for the Hemodynamics in fMRI Analysis". In: arXiv preprint arXiv:1708.06152 (2017).

[126] Horacio S Wio. Path integrals for stochastic processes: An introduction. World Scientific, 2013.

[127] John Wishart. "The generalised product moment distribution in samples from a normal multivariate population". In: Biometrika (1928), pp. $32-52$.

[128] H Rodney Withers. "The four R's of radiotherapy". In: Adv Radiat Biol 5.3 (1975), pp. 241-271.

[129] Ying Xiao, Lech Papiez, Rebecca Paulus, Robert Timmerman, William L Straube, Walter R Bosch, Jeff Michalski, and James M Galvin. "Dosimetric evaluation of heterogeneity corrections for RTOG 0236: Stereotactic body radiotherapy of inoperable stage I-II non-small-cell lung cancer". In: International Journal of Radiation Oncology* Biology* Physics 73.4 (2009), pp. 1235-1242.

[130] Cem Yolcu and Evren Özarslan. "Diffusion-weighted magnetic resonance signal for general gradient waveforms: multiple correlation function framework, path integrals, and parallels between them". In: Visualization and Processing of Higher Order Descriptors for Multi-Valued Data. Springer, 2015, pp. 3-19. 


\section{Papers}

The papers associated with this thesis have been removed for copyright reasons. For more details about these see:

http://urn.kb.se/resolve?urn=urn:nbn:se:liu:diva-1443.51 